\title{
Amplify-and-Forward Relaying with Maximal Ratio Combining over Fluctuating Two-Ray Channel: Non-Asymptotic and Asymptotic Performance Analysis
}

\author{
Hadi Hashemi, Javad Haghighat, Mohsen Eslami, and Keivan Navaie
}

\begin{abstract}
Fluctuating two-ray (FTR) channel model was shown to effectively characterize millimeter wave (mmWave) communication channels. In this paper, we adopt FTR to investigate amplify-and-Forward (AF) $\mathrm{mmWave}$ relaying system. Two communications scenarios are considered corresponding to the presence and absence of a direct link between the transmitter and receiver. Outage probability and symbol error rate (SER) are then analytically obtained as performance metrics. The results are further compared with the corresponding metrics obtained based on conventional channel models including Nakagami$m$ and two-wave with diffuse power (TWDP). Especially, for the high-SNR regime, our analyses indicate that performance evaluations based on the conventional models significantly deviate from that of based on the FTR model. Our results provide quantitative insights on the importance of model selection in design and performance evaluations of relay-based mmWave systems. Moreover, for the high-SNR regime, we carry out asymptotic analysis and obtain a low-complexity expression for the achieved AF relaying gain. Such an expression provides a quantitative measure on whether or not AF relaying outperforms no-relaying in a given setting. Extensive numerical and simulation results are provided to confirm the accuracy of the analysis and investigate system performance in different settings.
\end{abstract}

Index Terms-Amplify-and-Forward Relaying, High-SNR Regime, Fluctuating Two-Ray Channel, mmWave Communications.

\section{INTRODUCTION}

Millimeter wave (mmWave) communications is one of the promising solutions to the spectrum scarcity problem in the emerging new generations of cellular networks [1]. Nevertheless, the radio coverage in mmWave band is limited due to its inherent severe penetration loss and scattering effects. Relayaided communications is used to extend the radio coverage in this frequency band, see, e.g., [2]-[12] and references therein. In most of these works however, conventional fading channel models such as Rayleigh, Nakagami- $m$, and two-wave with diffuse power (TWDP) [13], [14] are considered for characterizing the mmWave channel. Such models however, are unable to fully characterize mmWave communications channels [15]. For example, the authors in [16] compare the experimentally measured Level Crossing Rates (LCRs)

H. Hashemi, J. Haghighat and M. Eslami are with Department of Electrical Engineering, Shiraz University of Technology, Shiraz, Iran (e-mail: \{h.hashemi, haghighat, m.eslami\}@sutech.ac.ir).

K. Navaie is with School of Computing and Communications, Lancaster University, UK (e-mail: k.navaie@lancaster.ac.uk) of mmWave channels with the simulated values found by applying different channel models. They then conclude that the Rayleigh channel model, and also in most scenarios the Rician channel model, are unable to closely track the measured LCRs, thus may not be proper fits to model signal propagation in mmWave bands. In order to effectively characterizing mmWave channel behavior, several modified channel models are proposed in [15], [17], [18], including a Fluctuating TwoRay (FTR) model [15] which is shown to closely follow the experimental data, especially in the $28 \mathrm{GHz}$ band.

In this paper, we analyze relay-aided communication over FTR channel. We focus on Amplify-and-Forward (AF) relaying schemes. In the following, we first review previous work on AF relaying, both in general and for mmWave communication. Then we address the challenges imposed by analyzing the system under FTR channel assumptions, and also clarify our contributions in this paper.

\section{A. Review of Previous Work}

Amplify-and-Forward (AF) relaying is a relaying technique, where the relay amplifies the received (analog) signal by applying either a fixed or a variable amplification gain and forwards it to the destination (or the next hop in multi-hop schemes). No attempt is made to decode the received signal (unlike Decode-and-Forward (DF) relaying) nor to compress it (unlike Compress-and-Forward (CF) relaying). As a results, AF relaying offers several advantages over DF and CF relaying, including a lower system and computational complexity, shorter delays, and a lower risk of breaching data security [19]-[21].

AF relaying for different applications in Microwave communication band is studied in several previous works, including [22]-[30]. In [26] the authors study multi-hop AF relaying schemes and derive the condition under which a multi-hop scheme outperforms a single-hop scheme. Furthermore, they introduce a design criterion which improves the system performance. In [25] the authors study how a delay in feeding back the estimated Channel State Information (CSI) from receiver to transmitter, will affect the performance of beamforming techniques applied to AF relaying schemes. They further derive exact as well as asymptotic expressions for system Bit Error Rate (BER) and outage probability, and show that feedback delay severely degrades the effectiveness of beamforming for AF relaying. 
In [24] the authors investigate the performance of antenna selection algorithms in AF relaying. They derive expressions for BER and outage probability, applying an optimal as well as a suboptimal antenna selection method. They show that for a sufficiently high Signal to Noise Ratio (SNR), the suboptimal scheme succeeds to perform close to optimal, while enjoying a smaller signaling overhead. They further conclude that for high-SNR regimes, their proposed suboptimal method is a suitable candidate for AF relaying schemes. In [23], [29], [30] the authors study AF relaying at presence of interference. In [23], the authors assume that signals transmitted by several co-channel interferers, interfere with the intended signal received at the relay. Assuming Rayleigh faded interference channels, BER and outage probability expressions are analytically derived and verified by Monte-Carlo simulations. A more complicated interference model is studied in [30]. Bounds on performance metrics are derived and the impacts of interference model parameters, including density of interfering nodes, on the system performance are studied. Authors in [29] consider the simultaneous effect of co-channel interference and channel estimation errors on the performance of interferencelimited AF relaying schemes.

In [27] the authors study Two-way AF relaying with imperfect self-interference cancellation. In addition to deriving several performance metrics, they introduce effective power allocation strategies that successfully compensate for the imperfect self-interference cancellation and reduce outage probability or BER of the system. In [22] the authors study AF relaying over time-selective fading channels, representing scenarios where at least one of the nodes in the network is mobile. Their derivations show that although in such cases the system experiences performance degradation, the degradation is less significant in scenarios where only the relay node is mobile while the source and destination nodes are static. Furthermore, performance of full-duplex AF relaying is analyzed and compared with the half-duplex relaying in [28].

$\mathrm{Up}$ on the introduction of mmWave communication as one of the key enabling technologies for $5 \mathrm{G}$, relaying came to immediate attention in order to assist mmWave schemes by extending their communication range and coverage. As noted previously, in mmWave band the penetration loss and scattering effects substantially increase compared to conventionally employed frequency bands for wireless networks, i.e. $6 \mathrm{GHz}$ and lower. Therefore, mmWave communication is naturally more suitable for short-range communication, unless assisted by a complementary technique, e.g. relaying. Subsequently, several recent works including [2]-[12] focus on the application of relays for mmWave communication, out of which [3], [8], [9], [11] explicitly consider AF relaying.

In [3] the authors consider the design of optimal beamforming algorithms for secure Multiple-Input Multiple-Output (MIMO) AF relaying schemes. Their proposed algorithm is shown to significantly increase the secrecy rate compared with other benchmark algorithms. Performance of multi-hop AF relaying is studied also in [8]. The multi-hop scheme is expected to overcome the blockage problem in mmWave, typically caused by buildings or similar obstacle. System BER is derived and optimal power allocation strategies are introduced in order to minimize the BER and optimize the performance. Authors in [9] derive conditions under which, one may decide whether relaying should be preferred to direct transmission, by looking at the beamwidth of employed directional antennas and the available power budget. Both half-duplex and fullduplex AF relaying schemes are studied. The authors also conclude that ground reflection has a significant impact on the system performance and should be carefully considered for designing efficient mmWave communication schemes. In [11] the authors study AF relaying with energy harvesting at the relay. They study the effect of energy harvesting parameters on outage probability and BER of the system.

\section{B. Motivation and Contributions}

As mentioned previously, most of recent works on relayaided mmWave communication, apply conventional channel models, including Rayleigh, Rician, and Nakagami-m. For example, the mmWave fading channels in [8] are modeled as Nakagami channels. However, recent empirical studies [18] reveal that the Probability Density Function (PDF) of the received power in mmWave band, exhibits a bimodality which may not be captured by aforementioned models. Consequently, the actual behavior of mmWave channels may not be accurately modeled by conventional channel models such as Nakagami-m model. For example, Fig. 10 shows a significant deviation between the behavior of Nakagami-m model and actual mmWave channel (drawn from empirical data). This implies that analyzing mmWave communication schemes by Nakagami-m or other traditional channel models may lead to oversimplification which results in sever deviation from reality and in some cases fails to provide constructive insight into the system behavior in practice.

In order to address the bimodal behavior of the mmWave channel, the TWDP model was proposed in [13]. The TWDP channel model successfully captures the bimodality of the PDF by considering two strongest waves received through the multipath fading environment. However the model is difficult to analyze and also considers constant-amplitude specular components. In practice, the specular components of the multipath fading channel are better modeled by random variables. Hence, the authors in [15] extend the TWDP model by adding a random coefficient to the specular components and reach the FTR channel model. In other words, FTR channel model may be considered as an evolved TWDP model which provides extra insight and additional flexibility, by allowing random changes of the amplitudes of the two strongest paths. This extra flexibility enables the FTR channel to more closely track the actual mmWave channel behavior. For example, one may observe in Fig. 10 that both TWDP and FTR models behave close to the empirical mmWave environment results; however, the FTR model is more successful in capturing the actual characteristics of the distribution function of the empirical data (see Fig. 10 and its corresponding discussions for more details).

The complex baseband received voltage for an FTR channel is represented as follows:

$$
V_{r}=\sqrt{\zeta} \cdot V_{1} \cdot \exp \left(j \phi_{1}\right)+\sqrt{\zeta} \cdot V_{2} \cdot \exp \left(j \phi_{2}\right)+X+j Y,
$$


where $\sqrt{\zeta}$ has Nakagami-m distribution, $\phi_{1}, \phi_{2}$ are independent and uniformly distributed random variables (r.v.), $X, Y$ are independent and normally distributed r.v., and $V_{1}, V_{2}$ are constant amplitudes. The FTR model includes several other well-known models as its special cases; e.g. for $V_{1}=V_{2}=0$ it reduces to Rayleigh channel model (see [15]-Table I, for details). The FTR model is widely adopted in recent works as an effective mmWave channel model in analyzing different communication schemes, see, e.g., [11], [12], [31]-[38] and references therein.

In this paper, we focus on AF relaying over FTR channels. In order to explain the novelty of the work, in the following, we study some of the relevant works on FTR channel and clarify the differences between those works and the work carried out in this paper.

In [12] the authors consider DF relaying over the FTR channel. Analytical treatment of DF relaying is fundamentally different from $\mathrm{AF}$ relaying. In DF relaying, the relay first decodes the signal received from the source; then, if the decoding is error-free, re-encodes and forwards the signal to the destination. Therefore, the source-relay and relay-destination channels may be treated as disjoint channels. End-to-end transmission from the source to destination is successful, if both source-relay and relay-destination channel SNRs are above a threshold required to guarantee error-free decoding. This corresponds to the case where the minimum of sourcerelay and relay-destination channel SNRs is above threshold. In summary, in order to analyze the DF relaying, PDF of the minimum of the two channel SNRs must be derived. However, in AF relaying, the end-to-end SNR is (approximately) the harmonic mean of the SNRs of source-relay and relay-destination channels. Therefore, in order to analyze $\mathrm{AF}$ relaying, derivation of the PDF of the harmonic mean of the two channel SNRs is required, that is a considerably more challenging problem in case of the FTR channels.

Notably, this problem is previously tackled in [11] and expressions for system performance metrics are derived. The focus in [11] is mostly on the effect of energy harvesting parameters on the system performance. However, the complicated nature of the derivations in [11] do not provide direct insight on how the performance metrics will change by changing the channel SNR and subsequently do not allow to conclude in which scenarios AF relaying outperforms pointto-point transmission.

In our paper, we derive clear equations to further investigate system performance and relaying gain in various SNR regions. In addition to the relay link, we consider the case where the destination also receives the signal through the direct (pointto-point) link and analyze the performance of Maximal Ratio Combining (MRC) in such scenarios. We further extend our derivations to asymptotic cases where the SNR values are sufficiently large, and derive low-complexity easy to interpret expressions for outage probability and Symbol Error Rate (SER).

Our asymptotic results enable us to address the question whether given a specific set of channel parameters, AF relaying outperforms point-to-point communication. It is also worth mentioning that although the works of [32] and [31] do not directly address relaying and instead focus on MRC an Equal Gain Combining (EGC), the results of [32] and [31] are applicable to DF relaying, as follows. If the relay decodes the source signal error-free and transmits to the destination, and meanwhile the destination receives another copy of the source signal via the direct (source to destination) link, the destination may combine the two received copies in order to gain better performance. Therefore, here we also compare the analysis of MRC and EGC combining techniques with the analysis carried out in this paper. As pointed out above, in $\mathrm{AF}$ relaying analysis, the goal is to derive the PDF of the harmonic mean of two channel SNRs. However, in MRC, the goal is different, namely it is to derive the PDF of summation of several channel SNRs [32]. The EGC problem addresses derivation of the PDF of summation of two or more channel gain amplitudes [31]; that is more challenging compared to MRC, but is still different from and less challenging than the problem of finding the PDF of the harmonic mean which is required for $\mathrm{AF}$ relaying analysis and is addressed in this paper.

Motivated by above discussions, in this paper we study the problem of AF relaying over FTR channels for the following two communications scenarios (see, Fig. 1). In the first scenario, communication is solely carried out via the relay, i.e., there is no direct link between source and receiver; whereas in the second scenario, the direct link is also present and MRC is adopted at the receiver to exploit the diversity gain. Our contributions are summarized as follows:

(i) We derive the PDF of the SNR at destination node, for both above-mentioned scenarios. Then we derive analytical expressions for the outage probability and SER of the system. Due to highly complex nature of the FTR channel model compared to previously applied models, an in-depth analysis is applied in order to obtain the derivations. However, the advantage of the derived equations is that they lend themselves to asymptotic analysis which provide clear new insights on the system performance.

(ii) We study the high-SNR regime and derive simplified asymptotic equations for system SER. Then we derive the gain of AF relaying compared to point-to-point communication in high-SNR region. The derived gain enables a central node (e.g., destination) to decide whether cooperation of a candidate relay is beneficial. The decision is made by calculating the asymptotic gain by plugging in the channel parameters and deciding to employ the relay only if the gain is larger than one (otherwise, the direct link is preferred). The derived asymptotic gain may also help with relay selection. If several candidate relays are available, the gain term for each of them may be calculated and the relay providing the largest gain is selected.

(iii) We provide extensive numerical results to verify the accuracy of our analytical derivations and also study the effects of different parameters on the system performance. Our simulations confirm the accuracy of the analysis and also clarify the impact of the adopted channel model on the accuracy of performance analysis. In particular, our results indicate that the performance evaluations based on commonly applied channel models for mm-Wave communications, e.g., Nakagami- $m$ and TWDP, may significantly deviate from reality, especially in 
high-SNR regime. This implies that designing a mmWave relying system based on the conventional channel model may in practice result in a much lower system performance than expected. Computational complexity of derived equations as well as the effect of power allocation on the system performance are also studied throughout our results.

The rest of this paper is organized as follows. In Section II, we present the system model. Section III presents our analyses to obtain the SNR distribution at the receiver. Then in Sections IV and V, we obtain outage probability and SER. Derivations for asymptotic cases, i.e. high-SNR regime, are carried out in Section V-A. Finally, Section VI presents our numerical results followed by conclusions drawn in Section VII.

\section{SySTEM MODEL}

We consider a relay-aided mmWave communications system (see, Fig. 1). In our model, relaying is used in cases where line-of-sight link between the receiver and the transmitter is either weak or completely blocked. The total energy budget per symbol transmission, $E_{s}$, is also equally divided between the transmitter and the AF relay, whereas in case of point-topoint communication, the whole energy budget is allocated to the source transmitter. For brevity, we define $\bar{\rho} \triangleq \frac{E_{s}}{N_{0}}$, where $\frac{N_{0}}{2}$ is the power spectral density of the additive white Gaussian noise (AWGN).

All channels are experiencing block-fading and are modeled as FTR channels. The FTR channel model is defined by three parameters $m, K, \Delta$, [15], where $m>0.5$ is the Nakagami-m fading figure corresponding to the random variable $\sqrt{\zeta}$ in (1), and $K, \Delta$ are defined as follows:

$$
\begin{aligned}
& K=\frac{V_{1}^{2}+V_{2}^{2}}{2 \sigma^{2}} \\
& \Delta=\frac{2 V_{1} V_{2}}{V_{1}^{2}+V_{2}^{2}}
\end{aligned}
$$

where $V_{1}, V_{2}$ are defined in (1) and $\sigma^{2}$ is the variance of zeromean, normally distributed random variables $X, Y$ in (1). In words, parameter $K$ quantifies the ratio of the sum of power gains of the two strongest paths to the sum of power gains of the rest of paths. Cumulative power of the rest of the paths is expressed as $2 \sigma^{2}$. Parameter $\Delta$ quantifies the closeness of the power levels received through the two strongest paths [15]. The channel power gain is also assumed to be normalized to one, i.e.

$$
\sigma^{2} \triangleq \frac{1}{2(K+1)}
$$

We further define the channel instantaneous SNR of sourcerelay, relay-destination, and source-destination links as $\rho_{1}=$ $\left|h_{\mathrm{SR}}\right|^{2} \times \frac{\bar{\rho}}{2}, \rho_{2}=\left|h_{\mathrm{RD}}\right|^{2} \times \frac{\bar{\rho}}{2}$ and $\rho_{3}=\left|h_{\mathrm{SD}}\right|^{2} \times \frac{\bar{\rho}}{2}$, respectively, where $\frac{\bar{\rho}}{2}$ is to represent equal energy allocation to the links. According to [39] the probability density function (PDF) of the SNR for each link is

$$
f_{\rho_{l}}(x)=\sum_{j_{l}=0}^{J_{\max }} \frac{A_{l}\left(j_{l}\right)}{(\bar{\rho} / 2)^{j_{l}+1}} x^{j_{l}} e^{-\frac{x}{\sigma^{2} \bar{\rho}}},
$$

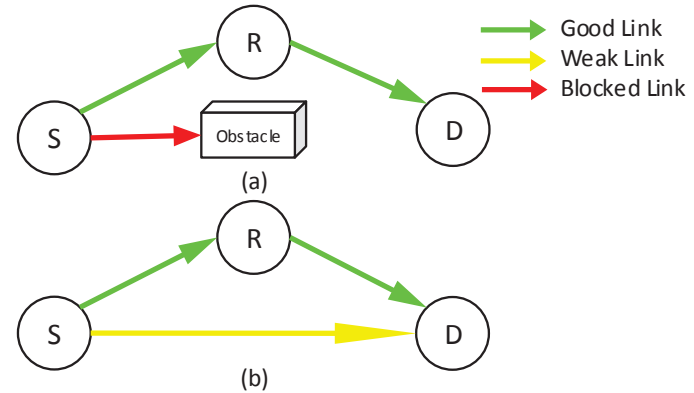

Fig. 1. Relay-aided AF system model with FTR channel.

where $l=1,2,3$, indicate the channel type, i.e., source-relay, relay-destination, and source-destination links, respectively. In the above, $J_{\max }$ is determined so that $\int f_{\rho_{l}}(x) d x \rightarrow 1$. In [39], it is shown that this condition holds for values of $J_{\max }$ as small as 40 . Further in (5), $A_{l}\left(j_{l}\right)$ depends on the channel $l$ parameters, denoted by $m_{l}, K, \Delta_{l}$. It is shown in [39] that for channel $l$,

$$
\begin{aligned}
& A_{l}\left(j_{l}\right)=\sum_{w=0}^{j_{l}} \sum_{t=0}^{w} \frac{m_{l}^{m_{l}} K^{j_{l}}\left(\begin{array}{c}
j_{l} \\
w
\end{array}\right)\left(\begin{array}{c}
w \\
t
\end{array}\right)\left(\frac{\Delta_{l}}{2}\right)^{w}}{\Gamma^{2}\left(j_{l}+1\right)\left(2 \sigma^{2}\right)^{j_{l}+1} \Gamma\left(m_{l}\right)} \\
& \quad \times \Gamma\left(j_{l}+m_{l}+2 t-w\right)\left(\left(m_{l}+K\right)^{2}-\left(K \Delta_{l}\right)^{2}\right)^{-\frac{j_{l}+m_{l}}{2}} \\
& \quad \times e^{\frac{-\pi(2 t-w) i}{2}} \mathcal{P}_{j_{l}+m_{l}-1}^{w-2 t}\left(\frac{m_{l}+K}{\sqrt{\left(m_{l}+K\right)^{2}-\left(K \Delta_{l}\right)^{2}}}\right)
\end{aligned}
$$

where $\Gamma($.$) represents the gamma function and \mathcal{P}_{\nu}^{\mu}(x)$ is Legendre's function of the first kind. We allow possibly different values for channel parameters, $m_{l}, \Delta_{l}$, for $l=1,2,3$; however, parameter $K$ is assumed to be identical for all channels. The reason for this condition is to manage the complexity of our analysis, and is later explained in Remark 1.

The relay applies dynamic $\mathrm{AF}$ amplification gain of $\alpha_{A F}=$ $\sqrt{\frac{E_{s}}{E_{s}\left|h_{S R}\right|^{2}+N_{0}}}$. As it is shown in [40], in this case the endto-end SNR over the S-R-D link is easily approximated by the harmonic mean of $\rho_{1}, \rho_{2}$ :

$$
\rho_{\mathrm{AF}} \approx \frac{\rho_{1} \times \rho_{2}}{\rho_{1}+\rho_{2}} .
$$

Alternatively, as it is seen in Fig. 1.b, one may combine signals received through S-D and S-R-D links using an MRC receiver. In this case, the output SNR of the MRC receiver is:

$$
\rho_{\mathrm{MRC}}=\rho_{\mathrm{AF}}+\rho_{3} .
$$

For easy reference, some of the parameters used throughout our analysis are also listed in Table I.

\section{Probability Density Function of the SNR}

For brevity, we start with defining the following parameters:

$$
\begin{gathered}
\sum_{j_{1}, j_{2}, j_{3}=0}^{J_{\max }} \triangleq \sum_{j_{1}=0}^{J_{\max }} \sum_{j_{2}=0}^{J_{\max }} \sum_{j_{3}=0}^{J_{\max }}, \\
\sum_{n_{1}, n_{2}=0}^{j_{1}, j_{2}} \triangleq \sum_{n_{1}=0}^{j_{1}} \sum_{n_{2}=0}^{j_{2}},
\end{gathered}
$$


TABLE I

LIST OF THE PARAMETERS.

\begin{tabular}{|c|c|}
\hline Symbol & Definition \\
\hline$E_{s}$ & Total energy budget for source and relay nodes \\
\hline$N_{0}$ & One-sided AWGN power spectral density \\
\hline$\sigma^{2}$ & $\begin{array}{l}\text { FTR channel parameter corresponding to cumulative } \\
\text { power of non-dominant paths }\end{array}$ \\
\hline$\rho_{1}, \rho_{2}, \rho_{3}$ & $\begin{array}{l}\text { SNRs of source-relay, relay-destination, and source- } \\
\text { destination links }\end{array}$ \\
\hline$\tilde{\rho_{3}}$ & $\begin{array}{l}\text { SNR of source-destination link, where relay is not } \\
\text { active and all energy budget is dedicated to the } \\
\text { source }\end{array}$ \\
\hline $\bar{\rho}$ & Expected value of SNR, defined as $\bar{\rho}=\frac{E_{s}}{N_{0}}$ \\
\hline$\rho_{A F}$ & $\begin{array}{l}\text { End-to-end SNR of source-relay-destination link for } \\
\text { AF relaying }\end{array}$ \\
\hline$\rho_{M R C}$ & SNR at the output of an MRC receiver at destination \\
\hline$\left(m_{l}, K, \Delta_{l}\right)$ & $\begin{array}{l}\text { FTR channel parameters for channel number } l= \\
1,2,3\end{array}$ \\
\hline$J_{\max }$ & $\begin{array}{l}\text { Maximum number of terms in the PDF expression } \\
\text { for FTR channel SNR. } J_{\max }=\infty \text { provides an } \\
\text { exact value; however, } J_{\max }=40 \text { is sufficiently } \\
\text { accurate. }\end{array}$ \\
\hline$K_{\max }$ & $\begin{array}{l}\text { Number of terms for Taylor series expansion of } \\
\text { summation of two variables to the power } b \text {. For } \\
\text { integer } b, K_{\max }=b \text {; otherwise } K_{\max }=\infty \\
\text { provides an exact value whereas } K_{\max } \text { in range of } \\
100 \text { provides sufficient accuracy. }\end{array}$ \\
\hline
\end{tabular}

$$
\begin{gathered}
a \triangleq \frac{2 j_{1}+2 j_{2}-n_{1}-n_{2}+1}{2}, \\
b \triangleq \frac{n_{1}+n_{2}-1}{2}, \\
c \triangleq a+b-k+1,
\end{gathered}
$$

where $n_{1}, n_{2}$, and $k$ are later defined. Note that $c$ is always an integer, whereas $a, b$ is either an integers or half-integers.

Here, we derive closed-form expressions for PDF of $\rho_{\mathrm{AF}}$ and $\rho_{\text {MRC }}$. These are then employed in Section IV to derive the outage probability and the SER. We begin with deriving the PDF of $\rho_{\mathrm{AF}}$. We define two auxiliary random variables, $W \triangleq \rho_{1}+\rho_{2}$, and $Z \triangleq \rho_{1} \times \rho_{2}$. In Appendix A, we show that the joint probability density function of $Z, W$ are (see Appendix A for details):

$$
\begin{aligned}
f_{Z, W}(z, w)= & \sum_{j_{1}, j_{2}=0}^{J_{\max }} \frac{4 A_{1}\left(j_{1}\right) A_{2}\left(j_{2}\right)}{\bar{\rho}^{j_{1}+j_{2}+2}} \\
& \times\left(g_{j_{1}, j_{2}}(z, w)+g_{j_{2}, j_{1}}(z, w)\right),
\end{aligned}
$$

where

$$
\begin{aligned}
g_{j_{1}, j_{2}}(z, w) & =\frac{\left(w+\sqrt{w^{2}-4 z}\right)^{j_{1}}\left(w-\sqrt{w^{2}-4 z}\right)^{j_{2}}}{\sqrt{w^{2}-4 z}} \\
& \times e^{-\frac{w}{\sigma^{2} \bar{\rho}}} U_{-1}\left(w^{2}-4 z\right)
\end{aligned}
$$

and $U_{-1}($.$) represents the unit step function. As it is seen in$ (7), $\rho_{\mathrm{AF}}=\frac{Z}{W}$, therefore, the cumulative density function of $\rho_{\mathrm{AF}}$ is

$$
F_{\rho_{\mathrm{AF}}}(h)=\int_{0}^{\infty} \int_{0}^{h w} f_{Z, W}(z, w) d z d w .
$$

Taking derivative with respect to $h$ yields:

$$
f_{\rho_{\mathrm{AF}}}(h)=\int_{4 h}^{\infty} w \cdot f_{Z, W}(h w, w) d w,
$$

where we note that for $w<4 h, g(h w, w)<0$. We further define,

$$
G_{j_{1}, j_{2}}(h) \triangleq \int_{4 h}^{\infty} w \cdot g_{j_{1}, j_{2}}(h w, w) d w .
$$

By replacing (9) in (12), and applying (13),

$$
f_{\rho_{\mathrm{AF}}}(h)=\sum_{j_{1}, j_{2}=0}^{J_{\max }} \frac{4 A_{1}\left(j_{1}\right) A_{2}\left(j_{2}\right)}{\bar{\rho}^{j_{1}+j_{2}+2}}\left(G_{j_{1}, j_{2}}(h)+G_{j_{2}, j_{1}}(h)\right) \text {. }
$$

Now we use binomial expansions of $\left(w+\sqrt{w^{2}-4 z}\right)^{j_{1}}$ and $\left(w-\sqrt{w^{2}-4 z}\right)^{j_{2}}$ in (10), followed by substituting the resulting series in (13) to reach:

$$
\begin{aligned}
& G_{j_{1}, j_{2}}(h)=\sum_{n_{1}, n_{2}=0}^{j_{1}, j_{2}}\left(\begin{array}{l}
j_{1} \\
n_{1}
\end{array}\right)\left(\begin{array}{l}
j_{2} \\
n_{2}
\end{array}\right)(-1)^{n_{2}} \\
& \times \int_{4 h}^{\infty} w^{\frac{2 j_{1}+2 j_{2}-n_{1}-n_{2}+1}{2}}(w-4 h)^{\frac{n_{1}+n_{2}-1}{2}} e^{-\frac{w}{\sigma^{2} \bar{\rho}}} d w .
\end{aligned}
$$

Let us define:

$$
\begin{aligned}
\Theta_{a, b}(h) & =\int_{4 h}^{\infty} w^{a}(w-4 h)^{b} e^{-\frac{w}{\sigma^{2} \bar{\rho}}} d w \\
& =\int_{4 h}^{\infty} w^{a+b}\left(1-\frac{4 h}{w}\right)^{b} e^{-\frac{w}{\sigma^{2} \bar{\rho}}} d w
\end{aligned}
$$

and

$$
(q)_{k}=\left\{\begin{array}{cc}
1, & k=0, \\
q(q+1) \cdots(q+k-1), & k>0 .
\end{array}\right.
$$

Let us substitute $\left(1-\frac{4 h}{w}\right)^{b}$ in (16) by its Taylor series approximation, as follows:

$$
\left(1-\frac{4 h}{w}\right)^{b}=\sum_{k=0}^{K_{\max }} \frac{(-b)_{k}}{\Gamma(k)} \times\left(\frac{4 h}{w}\right)^{k} .
$$

For integer-valued $\mathrm{b}, K_{\max }=b$ is exact; whereas for noninteger valued b, $K_{\max }$ in range of 100 is sufficiently accurate. Now, we may re-write $\theta_{a, b}($.$) as follows$

$$
\begin{aligned}
\Theta_{a, b}(h) & =\int_{4 h}^{\infty} w^{a+b}\left(\sum_{k=0}^{K_{\max }} \frac{(-b)_{k}}{\Gamma(k+1)} \frac{(4 h)^{k}}{w^{k}}\right) e^{-\frac{w}{\sigma^{2} \bar{\rho}}} d w \\
& =\sum_{k=0}^{K_{\max }} \frac{(-b)_{k}(4 h)^{k}}{\Gamma(k+1)} \int_{4 h}^{\infty} w^{a+b-k} e^{-\frac{w}{\sigma^{2} \bar{\rho}}} d w .
\end{aligned}
$$

Noting that $\int x^{m} e^{-n x} d x=n^{-m-1} \times \Gamma(m+1, n x)$, we then write:

$$
\Theta_{a, b}(h)=\sum_{k=0}^{K_{\max }} \frac{(-b)_{k}\left(\sigma^{2} \bar{\rho}\right)^{c}}{(4 h)^{-k} \Gamma(k+1)} \Gamma\left(c, \frac{4 h}{\sigma^{2} \bar{\rho}}\right),
$$

where $c \triangleq a+b-k+1$, and $\Gamma(.,$.$) represents the upper incom-$ plete gamma function. Replacing (20) in (15) and inserting the result in (14), followed by straightforward mathematical 
manipulations, we obtain the following closed-form for PDF of $\rho_{\mathrm{AF}}$ :

$$
f_{\rho_{\mathrm{AF}}}(h)=\sum_{j_{1}, j_{2}=0}^{J_{\max }} \sum_{n_{1}, n_{2}=0}^{j_{1}, j_{2}} \sum_{k=0}^{K_{\max }} \frac{\Omega_{\mathrm{AF}}}{\bar{\rho}^{k+1}} h^{k} \Gamma\left(c, \frac{4 h}{\sigma^{2} \bar{\rho}}\right),
$$

where

$$
\begin{aligned}
\Omega_{\mathrm{AF}} & \triangleq\left(\begin{array}{c}
j_{1} \\
n_{1}
\end{array}\right)\left(\begin{array}{c}
j_{2} \\
n_{2}
\end{array}\right)\left((-1)^{n_{1}}+(-1)^{n_{2}}\right) \\
& \times A_{1}\left(j_{1}\right) \cdot A_{2}\left(j_{2}\right) \frac{4^{k+1}(-b)_{k} \sigma^{2 c}}{\Gamma(k+1)} .
\end{aligned}
$$

Remark 1. In Section II we assume identical values for $K$ across different channels. This condition is set to ensure a common denominator for the exponential term, $e^{-\frac{x}{\sigma^{2} \bar{p}}}$, in PDFs of $f_{\rho_{1}}(x), f_{\rho_{2}}(x)$ (Eq. (5)). Note that due to normalizing the channel gain powers, $\sigma^{2}$ is related to $K$ through (4). Therefore, letting identical $K$ is equivalent to having identical $\sigma^{2}$ for all channels. The common denominator in turn simplifies the expressions of the joint distributions $f_{\rho_{1} \rho_{2}}(.,$.$) ,$ $f_{\rho_{2} \rho_{1}}(.,$.$) in (48) and consequently leads to the compact form$ of (9) for $f_{Z, W}(.,$.$) . For the general case of different \sigma^{2}$ values, the derived expression for $f_{Z, W}(.,$.$) is not as compact,$ and replacing the result in (12) may not lead to a satisfactory solution.

\section{A. SNR Probability Density Function: MRC}

For the MRC receiver,

$$
f_{\rho_{\mathrm{MRC}}}(u)=f_{\rho_{3}}(u) * f_{\rho_{\mathrm{AF}}}(u),
$$

where $f_{\rho_{3}}(\cdot)$, and $*$ are the PDF of S-D channel SNR, and convolution operator, respectively.

By replacing (5) and (21) into (23) we obtain

$$
f_{\rho_{\mathrm{MRC}}}(u)=\sum_{j_{1}, j_{2}, j_{3}=0}^{J_{\max }} \sum_{n_{1}, n_{2}=0}^{j_{1}, j_{2}} \sum_{k=0}^{K_{\max }} \frac{2^{j_{3}+1} \Omega_{\mathrm{AF}} A_{3}\left(j_{3}\right)}{\bar{\rho}^{j_{3}+k+2}} m(u),
$$

where $m(u)$ is obtained after convolving $u^{j_{3}} e^{-\frac{u}{\sigma^{2} \bar{\rho}}}$ and $u^{k} \Gamma\left(c, \frac{4 u}{\sigma^{2} \bar{\rho}}\right)$ as follows.

$$
m(u)=e^{-\frac{u}{\sigma^{2} \bar{\rho}}} \int_{0}^{u}(u-x)^{j_{3}} x^{k} \Gamma\left(c, \frac{4 u}{\sigma^{2} \bar{\rho}}\right) e^{\frac{x}{\sigma^{2} \bar{\rho}}} d x .
$$

In (52), we derive a series representation for $m(u)$ (see, Appendix B for details). After replacing (52) in (25) we conclude:

$$
\begin{aligned}
& f_{\rho_{\mathrm{MRC}}}(u)=\sum_{j_{1}, j_{2}, j_{3}=0}^{J_{\max }} \sum_{n_{1}, n_{2}, n_{3}=0}^{j_{1}, j_{2}, j_{3}} \sum_{k=0}^{K_{\max }} \sum_{i=0}^{c-1} \frac{\Omega_{\mathrm{MRC}}}{\bar{\rho}^{j_{3}-n_{3}+1}} \\
& \times u^{j_{3}-n_{3}} e^{-\frac{u}{\sigma^{2} \bar{\rho}}} \gamma\left(n_{3}+k+i+1, \frac{3 u}{\sigma^{2} \bar{\rho}}\right),
\end{aligned}
$$

where $\gamma(\cdot, \cdot)$ is the lower incomplete Gamma function and $\Omega_{\text {MRC }}$ is expressed as follows,

$$
\begin{aligned}
& \Omega_{\mathrm{MRC}} \triangleq\left(\begin{array}{c}
j_{1} \\
n_{1}
\end{array}\right)\left(\begin{array}{c}
j_{2} \\
n_{2}
\end{array}\right)\left(\begin{array}{c}
j_{3} \\
n_{3}
\end{array}\right) \frac{(-1)^{n_{1}}+(-1)^{n_{2}}}{(-1)^{n_{3}}} \sigma^{2 c+2 n_{3}+2 k+2} \\
& \frac{2^{j_{3}+2 i+2 k+3} A_{1}\left(j_{1}\right) \cdot A_{2}\left(j_{2}\right) \cdot A_{3}\left(j_{3}\right)(-b)_{k} \Gamma(c)}{3^{n_{3}+k+i+1} \Gamma(i+1) \Gamma(k+1)}
\end{aligned}
$$

\section{Outage Probability}

The outage probability is defined as the probability that the channel SNR falls below a pre-defined threshold, $\rho_{\text {th }}$. For the point-to-point communication scenario, where no relaying is involved, the outage probability is simply derived as:

$$
\begin{aligned}
& P_{\mathcal{O}, \mathrm{SD}}\left(\rho_{\mathrm{th}}\right)=\operatorname{Pr}\left\{\tilde{\rho}_{3}<\rho_{\mathrm{th}}\right\}=\int_{0}^{\rho_{\mathrm{th}}} f_{\tilde{\rho}_{3}}(x) d x \\
& =\sum_{j_{3}=0}^{J_{\max }} A_{3}\left(j_{3}\right)\left(2 \sigma^{2}\right)^{j_{3}+1} \gamma\left(j_{3}+1, \frac{\rho_{\mathrm{th}}}{2 \sigma^{2} \bar{\rho}}\right),
\end{aligned}
$$

where $\tilde{\rho}_{3}=\bar{\rho} \times\left|h_{\mathrm{SD}}\right|^{2}$ represents the SNR for a point-to-point communication link. In the above, the available energy, $E_{s}$, is fully allocated to the source transmission, therefore, $\bar{\rho} / 2$ in (5) is replaced by $\bar{\rho}$.

If there is no direct link available and the signal is delivered solely through the relay link, the outage probability is the probability that $\rho_{\mathrm{AF}}<\rho_{\mathrm{th}}$. Using the PDF of $\rho_{\mathrm{AF}}$ in (21), we then obtain:

$$
\begin{aligned}
& P_{\mathcal{O}, \mathrm{AF}}\left(\rho_{\mathrm{th}}\right)=\operatorname{Pr}\left\{\rho_{\mathrm{AF}}<\rho_{\mathrm{th}}\right\}=\int_{0}^{\rho_{\mathrm{th}}} f_{\rho_{\mathrm{AF}}}(x) d x \\
& =\sum_{j_{1}, j_{2}=0}^{J_{\max }} \sum_{n_{1}, n_{2}=0}^{j_{1}, j_{2}} \sum_{k=0}^{K_{\max }} \frac{\Omega_{\mathrm{AF}}}{\bar{\rho}^{k+1}} \eta_{c, k}\left(\rho_{\mathrm{th}}\right),
\end{aligned}
$$

where,

$$
\eta_{c, k}\left(\rho_{\text {th }}\right) \triangleq \int_{0}^{\rho_{\text {th }}} x^{k} \Gamma\left(c, \frac{4 x}{\sigma^{2} \bar{\rho}}\right) d x .
$$

It is straightforward to show that:

$$
\begin{aligned}
\eta_{c, k}\left(\rho_{\mathrm{th}}\right) & =\frac{\left(\sigma^{2} \bar{\rho}\right)^{k+1}}{4^{k+1}(k+1)} \gamma\left(c+k+1, \frac{4 \rho_{\mathrm{th}}}{\sigma^{2} \bar{\rho}}\right) \\
& +\frac{\rho_{\mathrm{th}}^{k+1}}{k+1} \Gamma\left(c, \frac{4 \rho_{\mathrm{th}}}{\sigma^{2} \bar{\rho}}\right) .
\end{aligned}
$$

Similarly, for the MRC receiver, the outage probability is derived after obtaining the integral in $(26)$ over $\left(0, \rho_{\text {th }}\right)$ :

$$
\begin{aligned}
& P_{\mathcal{O}, \mathrm{MRC}}\left(\rho_{\mathrm{th}}\right)=\operatorname{Pr}\left\{\rho_{\mathrm{MRC}}<\rho_{\mathrm{th}}\right\} \\
& =\sum_{j_{1}, j_{2}, j_{3}=0}^{J_{\max }} \sum_{n_{1}, n_{2}, n_{3}=0}^{j_{1}, j_{2}, j_{3}} \sum_{k=0}^{K_{\max }} \sum_{i=0}^{c-1} \frac{\Omega_{\mathrm{MRC}}}{\bar{\rho}^{j_{3}-n_{3}+1}} \\
& \times \int_{0}^{\rho_{\mathrm{th}}} u^{j_{3}-n_{3}} e^{-\frac{u}{\sigma^{2} \bar{\rho}}} \gamma\left(n_{3}+k+i+1, \frac{3 u}{\sigma^{2} \bar{\rho}}\right) d u .
\end{aligned}
$$

Let us define

$\mathbf{M}\left(j_{3}-n_{3}, n_{3}+k+i+1, \frac{3 u}{\sigma^{2} \bar{\rho}}\right) \triangleq \int_{0}^{\rho_{\text {th }}} \frac{\gamma\left(n_{3}+k+i+1, \frac{3 u}{\sigma^{2} \bar{\rho}}\right)}{u^{n_{3}-j_{3}} e^{\frac{u}{\sigma^{2} \bar{\rho}}}} d u$.

In Appendix $\mathrm{C}$ we derive a series representation for (33). By replacing (56) in (32) we then obtain a series representation for $P_{\mathcal{O}, \mathrm{MRC}}\left(\rho_{\mathrm{th}}\right)$.

\section{Symbol ERror Probability}

The Symbol Error Rate (SER) is defined as the probability that the symbol demodulated at destination is not identical 
TABLE II

AF RELAYING GAIN COMPARED TO POINT-TO-POINT COMMUNICATION FOR $K=15$ AND $\Delta=0.5$.

\begin{tabular}{|c|c|c|c|}
\hline$m_{1}$ & $m_{2}$ & $m_{3}$ & $G_{\mathrm{AF}}(d B)$ \\
\hline \hline 5.5 & 5.5 & 0.5 & 16.7892 \\
\hline 3.0 & 3.0 & 0.5 & 11.3834 \\
\hline 3.0 & 3.0 & 1 & 7.1229 \\
\hline 1.0 & 1.0 & 0.5 & 2.1275 \\
\hline 1.0 & 0.5 & 0.5 & -0.5016 \\
\hline 1.0 & 1.0 & 1.0 & -2.1326 \\
\hline 0.5 & 0.5 & 1.0 & -6.3792 \\
\hline 1 & 1 & 5.5 & -17.0512 \\
\hline
\end{tabular}

to the symbol transmitted by the source. For M-PSK and MQAM, and given a fixed SNR value, $h$, the SER is approximated as [41]:

$$
P_{\mathcal{E}}(h) \approx \sum_{r=1}^{R} \alpha_{r} e^{-\beta_{r} h}
$$

where the number of terms, $R$, is 2 and 4 , for M-PSK, and MQAM, respectively, and the corresponding coefficients $\alpha_{r}, \beta_{r}$ are given in [41, Table I and II].

Let us define the average SER, $\bar{P}_{\mathcal{E}}$, as the expected value of $P_{\mathcal{E}}(h)$. Using (34),

$$
\bar{P}_{\mathcal{E}} \approx \begin{cases}\sum_{r=1}^{R} \alpha_{r} \mathbb{E}\left[e^{-\beta_{r} \tilde{\rho}_{3}}\right], & \text { for Point-to-Point } \\ \sum_{r=1}^{R} \alpha_{r} \mathbb{E}\left[e^{-\beta_{r} \rho_{\mathrm{AF}}}\right], & \text { for } \mathrm{AF} \\ \sum_{r=1}^{R} \alpha_{r} \mathbb{E}\left[e^{-\beta_{r} \rho_{\mathrm{MRC}}}\right], & \text { for MRC }\end{cases}
$$

where $\tilde{\rho}_{3} \triangleq \bar{\rho} \times\left|h_{\mathrm{SD}}\right|^{2}$ is defined to emphasis that in point-topoint mode, the whole energy, $E_{s}$, is allocated to the source node.

To obtain $\bar{P}_{\mathcal{E}}$, we derive expressions for $\mathbb{E}\left[e^{-\beta_{r} \cdot \tilde{\rho}_{3}}\right]$, $\mathbb{E}\left[e^{-\beta_{r} . \rho_{A F}}\right]$, and $\mathbb{E}\left[e^{-\beta_{r} \cdot \rho_{\mathrm{MRC}}}\right]$. Using (5) after replacing $\bar{\rho} / 2$ by $\bar{\rho}$, it is straightforward to show that:

$$
\mathbb{E}\left[e^{-\beta_{r} \tilde{\rho}_{3}}\right]=\sum_{j_{3}=0}^{J_{\max }} A_{3}\left(j_{3}\right)\left(\frac{2 \sigma^{2}}{1+2 \sigma^{2} \beta_{r} \bar{\rho}}\right)^{j_{3}+1} \Gamma\left(j_{3}+1\right) .
$$

Applying the PDF function of $\rho_{A F}$ to (21), we further obtain:

$$
\mathbb{E}\left[e^{-\beta_{r} . \rho_{A F}}\right]=\sum_{j_{1}, j_{2}=0}^{J_{\max }} \sum_{n_{1}, n_{2}=0}^{j_{1}, j_{2}} \sum_{k=0}^{K_{\max }} \frac{\Omega_{\mathrm{AF}}}{\bar{\rho}^{k+1}} \xi_{c}\left(\beta_{r}\right),
$$

where $c \triangleq a+b-k+1$, and

$$
\xi_{c}\left(\beta_{r}\right) \triangleq \int_{0}^{\infty} x^{k} e^{-\beta_{r} \cdot x} \Gamma\left(c, \frac{4 x}{\sigma^{2} \bar{\rho}}\right) d x .
$$

Applying the results of [42, eq. 6.455.1] and Euler's hypergeometric transformations [43] we then obtain:

$$
\begin{aligned}
& \xi_{c}\left(\beta_{r}\right)=\frac{\left(\sigma^{2} \bar{\rho}\right)^{k+1} \Gamma(k+c+1)}{(k+1)\left(\sigma^{2} \bar{\rho} \beta_{r}+4\right)^{k+1}} \\
& \times{ }_{2} F_{1}\left(k+1,1-c, k+2, \frac{\beta_{r} \sigma^{2} \bar{\rho}}{\beta_{r} \sigma^{2} \bar{\rho}+4}\right) .
\end{aligned}
$$

TABLE III

AF RELAYING GAIN COMPARED TO POINT-TO-POINT COMMUNICATION FOR $K=15$, AND $m_{1}=m_{2}=m_{3}=3$.

\begin{tabular}{|c|c|c|c|}
\hline$\Delta_{1}$ & $\Delta_{2}$ & $\Delta_{3}$ & $G_{\text {AF }}(d B)$ \\
\hline \hline 0.1 & 0.1 & 0.9 & 7.2180 \\
\hline 0.1 & 0.6 & 0.8 & 2.3846 \\
\hline 0.3 & 0.6 & 0.8 & 2.4883 \\
\hline 0.6 & 0.6 & 0.8 & 1.0867 \\
\hline 0.1 & 0.1 & 0.6 & 1.1518 \\
\hline 0.2 & 0.2 & 0.4 & -2.9480 \\
\hline 0.8 & 0.8 & 0.8 & -3.6234 \\
\hline 0.7 & 0.7 & 0.5 & -5.0772 \\
\hline
\end{tabular}

For an MRC receiver, we apply the PDF function of $\rho_{\mathrm{MRC}}$ in (26) followed by the result of [42, Eq 6.455.2], to obtain:

$$
\begin{aligned}
& \mathbb{E}\left[e^{-\beta_{r} \rho_{\mathrm{MRC}}}\right]=\sum_{j_{1}, j_{2}, j_{3}=0}^{J_{\max }} \sum_{n_{1}, n_{2}, n_{3}=0}^{j_{1}, j_{2}, j_{3}} \sum_{k=0}^{K_{\max }} \sum_{i=0}^{c-1} \frac{\Omega_{\mathrm{MRC}}}{\bar{\rho}^{j_{3}-n_{3}+1}} \\
& \times \int_{0}^{\infty} u^{j_{3}-n_{3}} e^{-\frac{1+\beta_{r} \sigma^{2} \bar{\rho}}{\sigma^{2} \bar{\rho}} u} \gamma\left(n_{3}+k+i+1, \frac{3 u}{\sigma^{2} \bar{\rho}}\right) d u \\
& =\sum_{j_{1}, j_{2}, j_{3}=0}^{J_{\max }} \sum_{n_{1}, n_{2}, n_{3}=0}^{j_{1}, j_{2}, j_{3}} \sum_{k=0}^{K_{\max }} \sum_{i=0}^{c-1} \Omega_{\mathrm{MRC}} \\
& \times \frac{3^{k+i+n_{3}+1} \sigma^{2 j_{3}-2 n_{3}+2} \Gamma\left(k+i+n_{3}+2\right)}{\left(k+i+n_{3}+1\right)\left(\sigma^{2} \bar{\rho} \beta_{r}+4\right)^{k+i+j_{3}+2}} \\
& \times{ }_{2} F_{1}\left(1, k+i+j_{3}+2, k+i+n_{3}+2, \frac{3}{\beta_{r} \sigma^{2} \bar{\rho}+4}\right) .
\end{aligned}
$$

\section{A. High-SNR Regime}

Here we investigate $\bar{P}_{\mathcal{E}}$ for no relaying (point-to-point communication) and AF relaying scenarios for sufficiently large $\bar{\rho}$, also referred to as high-SNR regime. Analyzing high SNR regime enables us to quantify the achieved gain of adopting AF relaying as compared to transmission without relaying. We further use the analysis of high-SNR regime to gain insights on whether incorporation of relaying in a given setting is beneficial or not.

For $\bar{\rho} \rightarrow \infty$, the term corresponding to $j_{3}=0$ in (36) becomes dominant. Therefore, for point-to-point communication (35) is reduced to

$$
\bar{P}_{\mathcal{E}-\mathrm{SD}} \approx A_{3}(0) \times\left(\sum_{r=1}^{R} \frac{\alpha_{r}}{\beta_{r}}\right) \times\left(\frac{1}{\bar{\rho}}\right),
$$

where for $\bar{\rho} \gg 1$. For AF relaying, where $\bar{\rho} \gg 1$, in (37) the terms corresponding to $k=0$ become dominant. Therefore, by noting that in (39), $k+c=a+b+1=j_{1}+j_{2}+1$, and after letting $\rho \rightarrow \infty$ in (39), we have:

$$
\begin{aligned}
\bar{P}_{\mathcal{E}-\mathrm{AF}} & \approx \sum_{j_{1}, j_{2}=0}^{J_{\max }} \sum_{n_{1}, n_{2}=0}^{j_{1}, j_{2}} 4\left(\begin{array}{l}
j_{1} \\
n_{1}
\end{array}\right)\left(\begin{array}{c}
j_{2} \\
n_{2}
\end{array}\right)\left((-1)^{n_{1}}+(-1)^{n_{2}}\right) \\
& \times A_{1}\left(j_{1}\right) \cdot A_{2}\left(j_{2}\right) \sigma^{2 a+2 b+2} \Gamma(a+b+2) \\
& \times{ }_{2} F_{1}(1,-a-b, 2,1) \times\left(\sum_{r=1}^{R} \frac{\alpha_{r}}{\beta_{r}}\right) \times\left(\frac{1}{\bar{\rho}}\right) .
\end{aligned}
$$




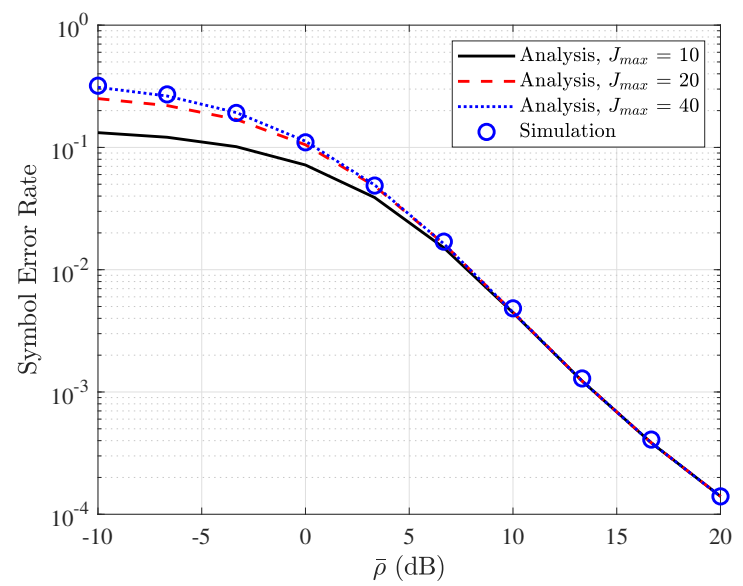

Fig. 2. The symbol error rate $v s . \bar{\rho}$ for direct link and different values of $J_{\max }$, where $K=15, m=5$ and $\Delta=0.5$.

We further note that ${ }_{2} F_{1}(1,-a-b, 2,1)=\frac{1}{a+b+1}$. Hence:

$$
\begin{aligned}
& \bar{P}_{\mathcal{E}-\mathrm{AF}} \approx \sum_{j_{1}, j_{2}=0}^{J_{\max }} \sum_{n_{1}, n_{2}=0}^{j_{1}, j_{2}} 4\left(\begin{array}{l}
j_{1} \\
n_{1}
\end{array}\right)\left(\begin{array}{l}
j_{2} \\
n_{2}
\end{array}\right) \frac{(-1)^{n_{1}}+(-1)^{n_{2}}}{\sigma^{-2 a-2 b-2}} \\
& \times A_{1}\left(j_{1}\right) \cdot A_{2}\left(j_{2}\right) \Gamma(a+b+1) \times\left(\sum_{r=1}^{R} \frac{\alpha_{r}}{\beta_{r}}\right) \times\left(\frac{1}{\bar{\rho}}\right) .
\end{aligned}
$$

Similarly, for the MRC receiver, the terms corresponding to $j_{3}=k=i=n_{3}=0$ in (40) become dominant. Therefore,

$$
\begin{aligned}
& \bar{P}_{\mathcal{E}-\mathrm{MRC}} \approx \sum_{r=1}^{R} \sum_{j_{1}, j_{2}=0}^{J_{\max }} \sum_{n_{1}, n_{2}=0}^{j_{1}, j_{2}} 8\left(\begin{array}{c}
j_{1} \\
n_{1}
\end{array}\right)\left(\begin{array}{c}
j_{2} \\
n_{2}
\end{array}\right) \\
& \times\left((-1)^{n_{1}}+(-1)^{n_{2}}\right) A_{1}\left(j_{1}\right) A_{2}\left(j_{2}\right) A_{3}(0) \\
& \times \Gamma\left(j_{1}+j_{2}+1\right) \sigma^{2 j_{1}+2 j_{2}+2} \frac{\alpha_{r}}{\beta_{r}^{2}}\left(\frac{1}{\bar{\rho}}\right)^{2} .
\end{aligned}
$$

From the derived asymptotic results, we then examine the asymptotic gain of $\mathrm{AF}$ relaying with respect to point-to-point communication. Let the SER of point-to-point communication, $P_{e}$, be equal to the SER achieved by AF relaying. Let $\bar{\rho}_{3}$ and $\bar{\rho}_{A F}$ represent the SNR values achieving $P_{e}$ in cases of point-to-point communication, and AF relaying, respectively. Replacing the left hand sides of (41) and (43) by $P_{e}$ followed by comparing the righthand sides of the two equations, leads to $\frac{\overline{\rho_{3}}}{\overline{\rho_{A F}}}=G_{A F}$, where ${ }^{1}$

$$
\begin{aligned}
G_{\mathrm{AF}}^{-1} & =\sum_{j_{1}, j_{2}=0}^{J_{\max }} \sum_{n_{1}, n_{2}=0}^{j_{1}, j_{2}} 2\left(\begin{array}{l}
j_{1} \\
n_{1}
\end{array}\right)\left(\begin{array}{l}
j_{2} \\
n_{2}
\end{array}\right)\left((-1)^{n_{1}}+(-1)^{n_{2}}\right) \\
& \times \frac{A_{1}\left(j_{1}\right) \cdot A_{2}\left(j_{2}\right)}{A_{3}(0)} \sigma^{2 j_{1}+2 j_{2}+2} \Gamma\left(j_{1}+j_{2}+1\right)
\end{aligned}
$$

\footnotetext{
${ }^{1}$ To obtain $G_{\mathrm{AF}}$, we further note that in no-relaying we dedicate the whole energy to the source, whereas in AF relaying the energy is equally divided between source and relay nodes. This is equivalent to assuming that the $\beta_{r}$ in $\mathrm{AF}$ mode is twice as high as the no relaying. The factor 2 is thus considered in deriving $G_{A F}$.
}

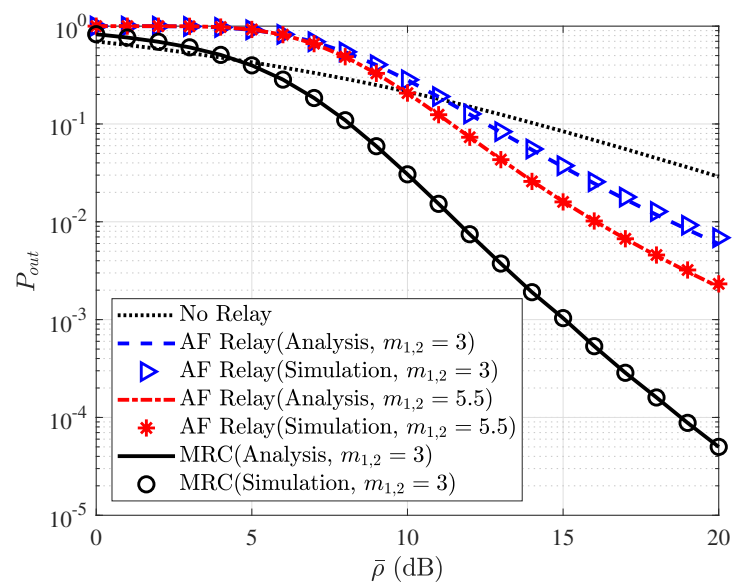

Fig. 3. The outage probability vs. $\bar{\rho}$ for the direct link, relay link, and MRC receiver, where $K=15, \Delta=0.5$, and $m_{3}=0.5$.

denotes the asymptotic gain of $\mathrm{AF}$ relaying compared to no-relaying and $G_{A F}>1$ represents scenarios, where $\mathrm{AF}$ relaying outperforms no-relaying.

In Table II, $G_{\mathrm{AF}}$ is given for different values of $m_{1}, m_{2}$, and $m_{3}$. It is observed that when the source-relay and relaydestination links are strong compared to the direct link, i.e., in cases where $m_{1}$ and $m_{2}$ are sufficiently large compared to $m_{3}$, AF relaying outperforms point-to-point communication in high-SNR regime. Table III presents $G_{A F}$ for $m_{l}^{\prime} s=3$, and different values of $\Delta_{l}$, where a larger $\Delta_{l}$ represents a more severe fading. When $\Delta_{1}$ and $\Delta_{2}$ are sufficiently smaller than $\Delta_{3}$, i.e. when the fading effect over source-relay and relaydestination links is less severe compared to the direct link, $\mathrm{AF}$ relaying introduces a gain over no-relaying. Otherwise, no-relaying is preferred and requires a smaller SNR value in order to achieve SER values identical to AF relaying.

\section{Numerical and Simulations Results}

Here we carry out simulations in order to evaluate the system performance and also verify the accuracy of our analysis. Monte Carlo simulations with $10^{7}$ transmitted symbols are applied. The channel gains are normalized by setting the expected value of their squares to 1 . The total energy budget is set to $E_{s}=1$, and $N_{0}$ is accordingly tuned to reach the desired SNR value $(\bar{\rho})$. The analytical results are plotted for $J_{\max }=40$ (which is the same value as recommended in [39]). To verify that this value of $J_{\max }$ in fact leads to sufficiently accurate results, in Fig. 2 we plot the SER of the point-topoint link (found by substituting (36) in (35)) for different values of $J_{\max }\left(J_{\max }=10,20,40\right)$ and compare the results with the simulated SER values. As it is seen, the results given by $J_{\max }=10$ substantially differ from the simulated values; whereas the results for $J_{\max }=20$, and $J_{\max }=40$ are looking fairly close, and almost identical to the simulated values, respectively. Therefore, $J_{\max }=40$ seems to provide a sufficient level of accuracy and will be applied to plot our analytical results throughout this section.

Fig. 3 shows the outage probability for direct link, relay link and output of MRC combiner. It is observed that for a large SNR, AF relaying outperforms point-to-point communication 


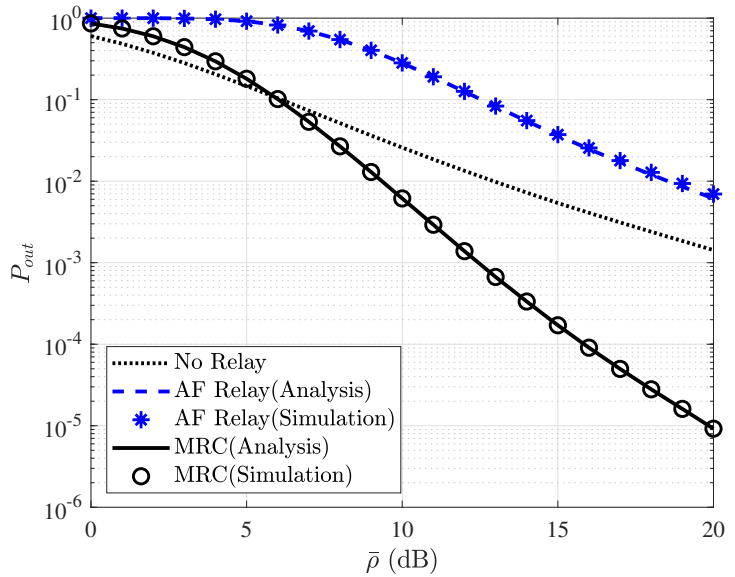

Fig. 4. The outage probability $v s . \bar{\rho}$ for the direct link, relay link, and MRC receiver, where $K=15, \Delta=0.5$, and $m_{1}=m_{2}=m_{3}=0.5$.

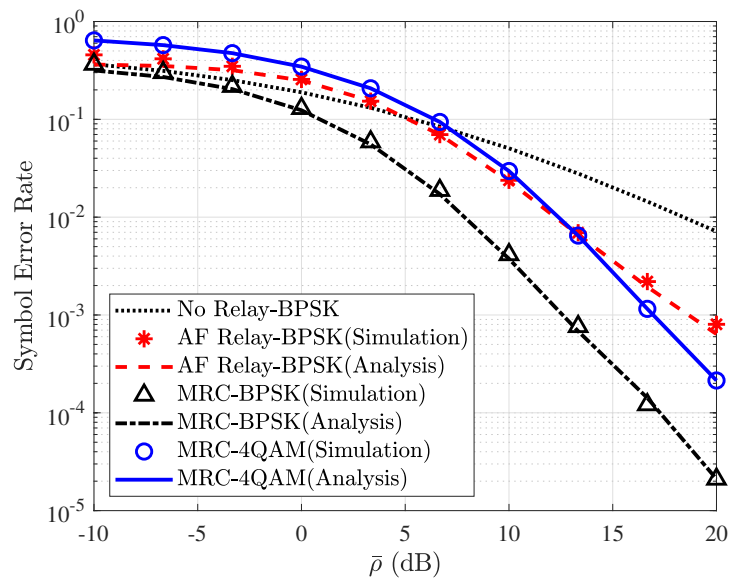

Fig. 5. The symbol error rate vs. $\bar{\rho}$ for direct link, relay link and MRC receiver, where $K=15, \Delta_{1}=\Delta_{2}=\Delta_{3}=0.5, m_{1}=m_{2}=3$, and $m_{3}=0.5$.

(i.e., no relays). Furthermore, by increasing the Nakagami- $m$ figures, $m_{1}, m_{2}$ from 3 to 5.5 , the performance of AF relaying improves as expected and an additional $2 \mathrm{~dB}$ gain at the outage probability of $10^{-2}$ is observed. Furthermore, for cases where the source-destination link is not blocked, (see, Fig. 1.b), MRC may be applied which offers an additional gain of $6 \mathrm{~dB}$ at the outage probability of $10^{-2}$.

Fig. 4 shows the outage probability of all scenarios for the Nakagami- $m$ figures, $m_{1}, m_{2}$ and $m_{3}$ all equal to 0.5 . As it is seen, the performance of $\mathrm{AF}$ relaying is inferior to that of no relaying. However, the MRC receiver may still take advantage of the relay link to improve the performance compared to norelaying. This improvement surpasses $8 \mathrm{~dB}$, at $P_{\mathcal{O}}=10^{-3}$.

Fig. 5 shows the symbol error rate of BPSK and 4-QAM versus $\bar{\rho}$ for scenarios where a direct S-D link is present. As it is seen, in high SNR regime, similar to the outage performance, AF relaying outperforms no-relaying. It is also seen that adopting MRC offers an additional performance gain. Fig. 6 shows the simulated values and the asymptotic analytical approximations for symbol error rate versus $\bar{\rho}$ for direct link (41), relay link (43) and MRC receiver (44). In order to study the asymptotic case which corresponds to large SNR values, we plot the asymptotic curves for the SNR values larger

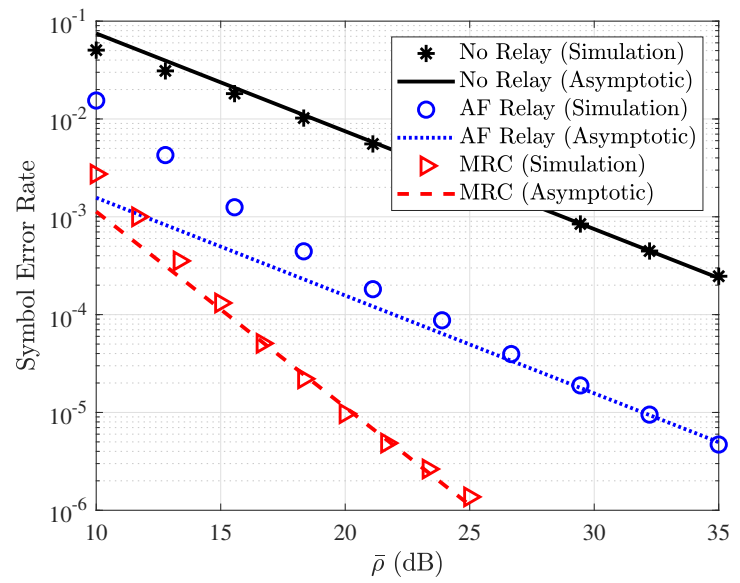

Fig. 6. Exact and asymptotic symbol error rate $v s . \bar{\rho}$ for direct link, relay link and MRC receiver, where $K=15, \Delta_{1}=\Delta_{2}=\Delta_{3}=0.5, m_{1}=$ $m_{2}=5.5$, and $m_{3}=0.5$.

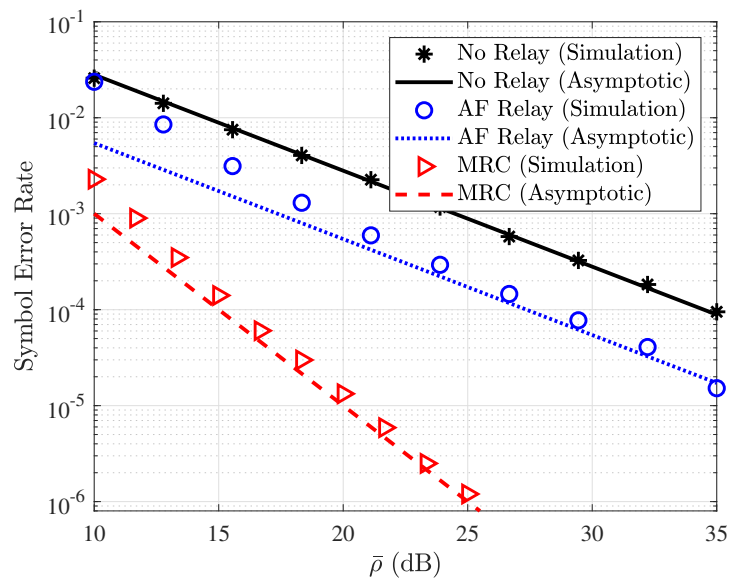

Fig. 7. Exact and asymptotic symbol error rate $v s . \bar{\rho}$ for direct link, relay link and MRC receiver, where $K=15, \Delta_{1}=\Delta_{2}=\Delta_{3}=0.5, m_{1}=m_{2}=3$, and $m_{3}=1$.

than $10 \mathrm{~dB}$. As it is seen, in cases where the expected value of SNR is greater than $20 \mathrm{~dB}$ the approximated asymptotic symbol error rate values are very close to simulation results. This observation suggests that at very high SNR values, where simulations are time consuming, one may directly apply the asymptotic derivations and expect to find SER values very close to reality. Fig. 7 illustrates asymptotic and simulation results for a different set of channel parameters. As observed, in both cases of Fig. 6, 7, the asymptotic gain of AF relaying with respect to no relaying, is identical to the gain values found in Table II.

Figure 8 illustrates the asymptotic and simulation results, where the channel parameters, $\Delta_{l}$, are set according to the first row of Table III. A larger value for $\Delta_{l}$ means that the corresponding channel is experiencing more severe fading [15]. Since in case of Fig. 8, $\Delta_{3}=0.9$ is considerably greater than $\Delta_{1}=\Delta_{2}=0.1$, it is expected that the direct link experience a considerably more severe fading compared to the relay link. The results of Fig. 8 confirm this conclusion and show that AF relaying outperforms point-to-point communication in this case. Moreover, comparing the curves at SER of $10^{-4}$ shows that $\mathrm{AF}$ relaying requires a SNR value which is around $7 \mathrm{~dB}$ 


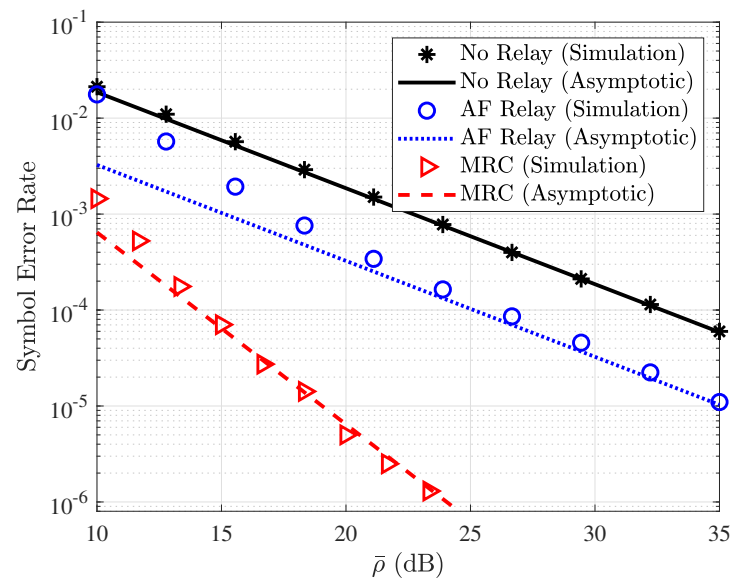

Fig. 8. Exact and asymptotic symbol error rate $v s . \bar{\rho}$ for direct link, relay link and MRC receiver, where $K=15, m_{1}=m_{2}=m_{3}=3, \Delta_{1}=\Delta_{2}=0.1$ and $\Delta_{3}=0.9$.

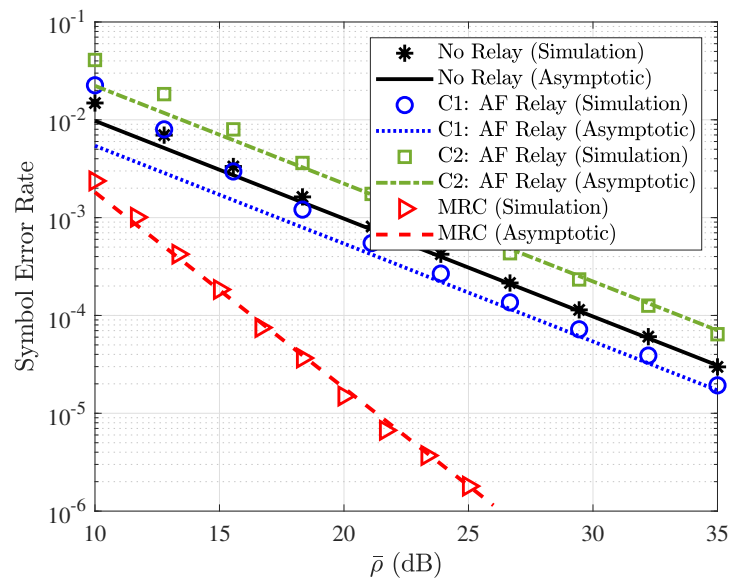

Fig. 9. Exact and asymptotic symbol error rate $v s . \bar{\rho}$ for direct link, relay link and MRC receiver, where $K=15, m_{1}=m_{2}=m_{3}=3$ and $\Delta_{3}=0.8$. For Case $1(\mathrm{C} 1): \Delta_{1}=0.1, \Delta_{2}=0.6$ and Case $2(\mathrm{C} 2): \Delta_{1}=\Delta_{2}=0.8$. The MRC curve is corresponding to $\mathrm{C} 2$.

smaller than that of no relaying. This observation is consistent with the asymptotic gain of AF relaying over no relaying, denoted in the first row of Table III. Asymptotic results for varying $\Delta_{l}$ 's are presented in Fig. 9. Two cases are studied, where AF relaying is and is not beneficial. As it is seen, even in the second case, applying the MRC receiver still provides a significant gain with respect to no-relaying.

\section{A. Comparison with Other Channel Models}

In the following, we compare the FTR channel model with other channel models commonly studied in the context of mmWave communications. In Fig. 10 the experimentally measured Cumulative Density Function (CDF) values for the received power at $28 \mathrm{GHZ}$ [18] are plotted and compared with the CDF values found by applying different channel models. As it is seen, the FTR model is a better fit and provides values closer to reality. To be more precise, we measured the Kolmogorov-Smirnov distance (KS distance) between the experimental $\mathrm{CDF}$ and the CDFs obtained for different channel models. The KS distance is defined as

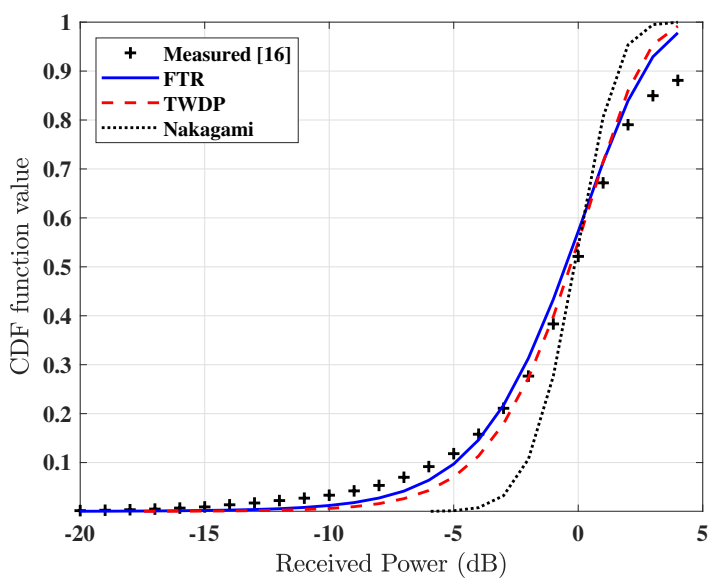

Fig. 10. Cumulative Density Function (CDF) of the received power for different channel models compare with measured $\mathrm{CDF}$ values at $28 \mathrm{GHz}$ ( [15], [18]), where $K=10, m=4$ and $\Delta=0.5$ (for TWDP model only parameters $K, \Delta$ are defined. For Nakagami-m model, only parameter $m$ is defined).

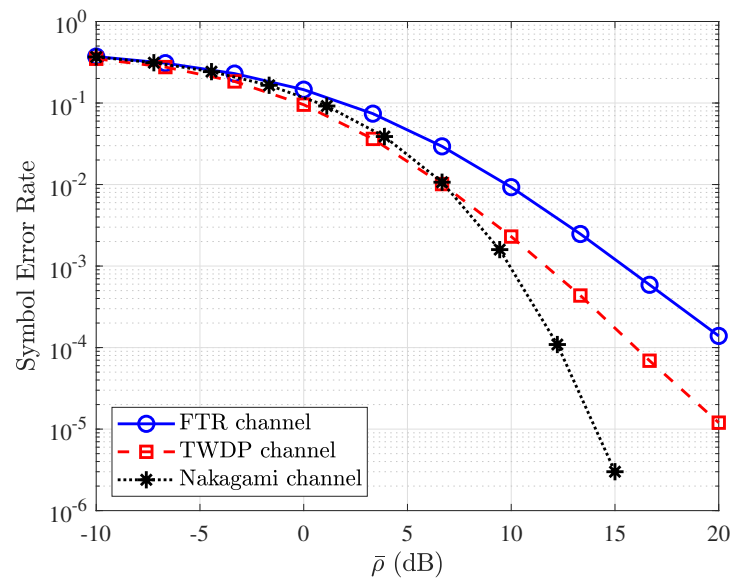

Fig. 11. The symbol error rate $v s . \bar{\rho}$ for MRC receiver with different channel models, where $K=15, m_{1}=m_{2}=m_{3}=3, \Delta_{1}=\Delta_{2}=0.5$, and $\Delta_{3}=0.8$.

the maximum absolute value of the difference between the two CDFs. The KS distances between the experimental CDF and the CDFs corresponding to Nakagami, TWDP, and FTR models are $0.1785,0.0403,0.0285$, respectively. Furthermore, the RMSEs between the empirical CDF values and the CDF values corresponding to Nakagami, TWDP and FTR channels are measured as $0.0864,0.0403,0.0334$, respectively. We note that the TWDP model also sounds convincingly close to reality. Nonetheless, the FTR model is more flexible and should provide a better fit due to incorporating an additional parameter that models the randomness of gain amplitudes for the two dominant paths (parameter $\sqrt{\zeta}$ in (1)).

Fig. 11 compares the SER corresponding to Nakagami$m$, TWDP, and FTR channel models. Since the FTR model was shown to more closely follow empirical results, one may conclude that both Nakagami- $m$ and TWDP provide results that are rather too optimistic-at least for the given set of channel parameters. This is more evident in case of Nakagami- $m$ channel; i.e., Nakagami- $m$ fails to accurately 


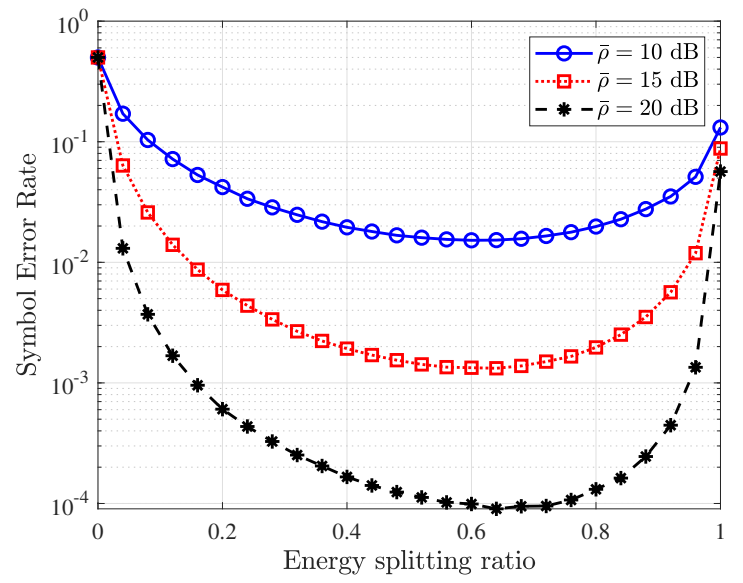

Fig. 12. The symbol error rate versus the energy splitting ratio, where $K=$ $15, \Delta_{1}=\Delta_{2}=\Delta_{3}=0.5$, and $m_{1}=m_{2}=m_{3}=5$.

model practical scenarios especially in high-SNR regimes. TWDP closely follows FTR, but still provides results which are about $3 \mathrm{~dB}$ too optimistic. For example, to achieve SER of $10^{-3}$, FTR predicts $15 \mathrm{~dB}$ required SNR; however, TWDP predicts a $12 \mathrm{~dB}$ required SNR value.

\section{B. Effect of power allocation}

Throughout this paper, we assumed that in presence of a relay, the energy budget, $E_{s}$, is equally divided between the source and the relay. A more effective approach is to solve a power allocation problem and find an optimal power allocation strategy for each given set of channel parameters. Although taking an analytical power allocation approach is out of the scope of this paper, we run a set of simulations to study the effect of power allocation on the system performance. For this, first let us define an energy splitting ratio, $0 \leq \lambda \leq 1$, where a given value of $\lambda$ indicates that the energy levels allocated to source and relay are $\lambda . E_{s}$, and $(1-\lambda) . E s$, respectively.

Figure 12 shows the SER versus the energy splitting ratio, $\lambda$, for different values of $\bar{\rho}$ (where $\bar{\rho}=\frac{E_{s}}{N_{0}}$ ). It is observed that for each value of $\bar{\rho}$, there exists an optimum energy-splitting ratio, $\lambda_{o p t}$, that minimizes the SER (assuming all other channel parameters are fixed to the values denoted in Fig. 12). The corresponding optimal values of $\lambda_{\text {opt }}$ for $\bar{\rho}=10,15,20 \mathrm{~dB}$ are $\lambda_{\text {opt }}=0.6,0.63,0.64$, respectively. It is also observed that for the example provided in Fig. 12, the suboptimal ratio of $\lambda=0.5$ leads to close-to-optimal results, i.e., the SER value at $\lambda=0.5$ is only slightly higher than the SER value provided by $\lambda_{\text {opt }}$.

\section{Computational complexity}

In previous sections, we pointed out that asymptotic derivations presented in Section V-A enjoy considerably smaller computational complexity, compared to the non-asymptotic derivations. To further elaborate on this point, let us consider a scenario, where a candidate relay is available to assist with the transmission. Further assume that all channel statistics are known and a central node (e.g., destination) aims to evaluate the gain of relay link compared to the direct link and prefers

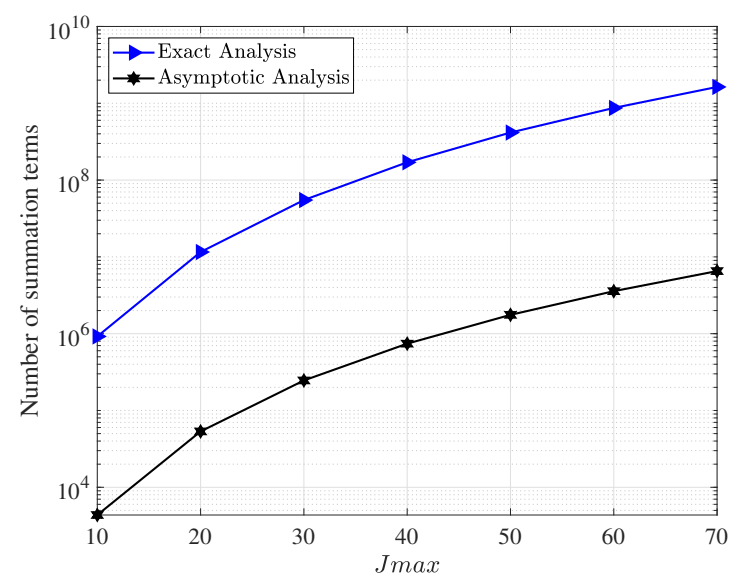

Fig. 13. Comparison between the number of summation terms required to calculate and compare the performance of the AF link with the point-topoint link, in cases of (i) Exact (non-asymptotic) analysis, and (ii) Asymptotic analysis.

the relay link over the direct link if the gain is positive (in $\mathrm{dB}$ scale, or greater than one in linear scale). The central node may take one of the following approaches: (i) Exact analysis: The central node evaluates $\mathbb{E}\left[e^{-\beta_{r} \tilde{\rho}_{3}}\right]$, and $\mathbb{E}\left[e^{-\beta_{r} \rho_{A F}}\right]$ by applying (36) and (37), respectively. Then it replaces the evaluated values in (35) to find the SER values for direct and relay links. The central node then compares the two obtained values and concludes whether applying the relay is beneficial. (ii) Asymptotic analysis: In high-SNR regime, the central node may instead evaluate $G_{A F}$ from (45) and decide to use the relay link if $G_{A F}>1$.

In Fig. 13 we compare the number of summation terms required to carry out each of the approaches (i) and (ii). For a non-integer $b$, we set $K_{\max }=100$. The results show that the asymptotic approach requires an impressively smaller number of summation terms. For example, at $J_{\max }=40$, the number of terms required for the exact and asymptotic approaches are $1.70 \times 10^{8}$ and $7.41 \times 10^{5}$, respectively; i.e., the asymptotic approach requires 230 times smaller number of summation terms. This is important when the system parameters vary quickly and long delays are not tolerable. In other words, if the channels' parameters vary before the central node completes its evaluation and reaches a decision, the decision is no longer valid for the updated channel parameters. The asymptotic approach is more likely to be successful in such scenarios, since it enjoys impressively smaller complexity and enables the central node to decide with considerably smaller delay. This is however under the condition that the expected value of SNR, $\bar{\rho}$, is sufficiently large to ensure the validity of applying an asymptotic analysis.

\section{Discussions}

In summary, our simulation results verified the accuracy of our analysis, in both non-asymptotic and asymptotic cases. Verifying the accuracy of asymptotic results suggests that at high-SNR region, where running Monte-Carlo simulations is very time-consuming, the low-complexity asymptotic equations may be employed to promptly evaluate the system 
performance with very high accuracy. For example, if one aims to find a proper design SNR value for achieving an SER of $10^{-10}$, and if counting 100 errors is required to evaluate the SER by simulations, then $10^{12}$ samples must be simulated for each SNR value. However, one may instead apply the asymptotic analytical results to plot the SER versus SNR curve and find the SNR value for which SER reaches $10^{-10}$.

Moreover, the asymptotic results are useful for relay selection. The asymptotic gain, $G_{A F}$, derived in (45) may be applied to verify whether for a given set of channel parameters, AF relaying outperforms point-to-point communication. If several candidate relays are available, the relay offering the highest gain may be selected. We further plotted the CDFs of different channel models and compared them with the CDF of experimental data. The plots confirmed that FTR channel model is a better fit to experimental data, compared to TWDP and Nakagami-m models. Then we compared the SER curves of the three models, and keeping in mind that FTR model more accurately reflects the reality of the channel behavior, we concluded that TWDP and Nakagami-m models make inaccurate predictions of the system SER. This result is important since it suggests that if only one channel parameter ( $m$ for Nakagami-m) or only two channel parameters $(K, \Delta$ for TWDP) are considered, a clear vision of the performance may not be gained.

We also carried out complexity studies and demonstrated that asymptotic analysis requires number of summation terms that are smaller than the ones required for non-asymptotic analysis by several orders of magnitude. Therefore, in delaysensitive systems where a central node needs a fast and low-complexity assessment method, the asymptotic equations are definitely favorable (at high-SNR region). Eventually, we studied the effect of power allocation and showed that although equal power allocation is suboptimal, it may perform close to optimal. This is interesting, since equal power allocation does not need an extra step of solving optimization problems (hence enjoying lower complexity and smaller delays).

\section{CONCLUSIONS}

We obtained closed-form expressions for the outage probability and symbol error rate performance in AF relaying in mmWave systems with FTR channels. In case of presence of a direct link, analysis was extended to include MRC receivers at destination. Asymptotic results were also derived and asymptotic gain of AF relaying compared to no-relaying was quantified. The results enables us to clearly answer the question whether or not, given a certain setting, AF relaying outperforms no-relaying. Our analytical and simulation results showed that the gain of $\mathrm{AF}$ relaying over no-relaying is significant, reaching $16 \mathrm{~dB}$ in certain scenarios. Furthermore, the simple and low-complexity expression derived for the asymptotic gain, can be used as an indicator to select the best relay in communication schemes where several potential nodes are available for relaying. Our results further showed that adopting conventionally applied channel models, e.g., TWDP and Nakagami-m models, for mm-Wave communications provides results that deviate from reality, especially for higher SNR values.

\section{APPENDIX A \\ JOINT PDF OF $Z, W$ IN (9)}

Based on the definition of variables $W$ and $Z$, we have two solutions for source-relay and relay-destination link SNRs as follows,

$$
\begin{aligned}
& \left(\rho_{1,1}, \rho_{2,1}\right)=\left(\frac{W+\sqrt{W^{2}-4 Z}}{2}, \frac{W-\sqrt{W^{2}-4 Z}}{2}\right), \\
& \left(\rho_{1,2}, \rho_{2,2}\right)=\left(\frac{W-\sqrt{W^{2}-4 Z}}{2}, \frac{W+\sqrt{W^{2}-4 Z}}{2}\right),
\end{aligned}
$$

where the second index represents the solution number 1 or 2.

Using the method of [44, Sec. 6.3], the Jacobians of the transformation as per $W$ and $Z$ are $J_{1}=\sqrt{W^{2}-4 Z}$ and $J_{2}=-\sqrt{W^{2}-4 Z}$. Therefore, the joint PDF of $W$ and $Z$ is

$$
f_{Z W}(z, w)=\frac{f_{\rho_{1} \rho_{2}}\left(\rho_{1,1}, \rho_{2,1}\right)}{\left|J_{1}\right|}+\frac{f_{\rho_{1} \rho_{2}}\left(\rho_{1,2}, \rho_{2,2}\right)}{\left|J_{2}\right|} .
$$

By substituting the Jacobians in (48), and also noting that $\rho_{1}, \rho_{2}$ are independent random variable with their PDF given in (5), it is straightforward to obtain (9).

\section{APPENDIX B}

SERIES REPRESENTATION OF $m(u)$ IN (25)

Replacing the binomial expansion of $(u-x)^{j_{3}}$ in (25) yields:

$$
\begin{aligned}
m(u) & =u^{j_{3}-n_{3}} e^{-\frac{u}{\sigma^{2} \bar{\rho}}} \sum_{n_{3}=0}^{j_{3}}\left(\begin{array}{c}
j_{3} \\
n_{3}
\end{array}\right)(-1)^{n_{3}} \\
& \times \int_{0}^{u} x^{n_{3}+k} \Gamma\left(c, \frac{4 u}{\sigma^{2} \bar{\rho}}\right) e^{\frac{x}{\sigma^{2} \bar{\rho}}} d x .
\end{aligned}
$$

We also note that $c$ is an integer, therefore the upper incomplete gamma function can be represented by a finite series as derived in [42, eq. 8.352.2]:

$$
\Gamma\left(c, \frac{4 u}{\sigma^{2} \bar{\rho}}\right)=\sum_{i=0}^{c-1} \frac{\Gamma(c)}{\Gamma(i+1)}\left(\frac{4 u}{\sigma^{2} \bar{\rho}}\right)^{i} e^{-\frac{4 u}{\sigma^{2} \bar{\rho}}} .
$$

We then insert ((50)) in (49) to obtain:

$$
\begin{aligned}
m(u) & =u^{j_{3}-n_{3}} e^{-\frac{u}{\sigma^{2} \bar{\rho}}} \sum_{n_{3}=0}^{j_{3}} \sum_{i=0}^{c-1}\left(\begin{array}{c}
j_{3} \\
n_{3}
\end{array}\right)(-1)^{n_{3}} \\
& \times \frac{\Gamma(c)}{\Gamma(i+1)}\left(\frac{4}{\sigma^{2} \bar{\rho}}\right)^{i} \int_{0}^{u} x^{n_{3}+k+i} e^{-\frac{3 x}{\sigma^{2} \bar{\rho}}} d x .
\end{aligned}
$$

Straightforward integration of $\int_{0}^{u} x^{n_{3}+k+i} e^{-\frac{3 x}{\sigma^{2} \bar{\rho}}} d x$ gives the following series representation for $m(u)$.

$$
\begin{aligned}
m(u) & =\sum_{n_{3}=0}^{j_{3}} \sum_{i=0}^{c-1}\left(\begin{array}{c}
j_{3} \\
n_{3}
\end{array}\right)(-1)^{n_{3}} \frac{4^{i} \Gamma(c)\left(\sigma^{2} \bar{\rho}\right)^{n_{3}+k+1}}{3^{n_{3}+k+i+1} \Gamma(i+1)} \\
& \times u^{j_{3}-n_{3}} e^{-\frac{u}{\sigma^{2} \bar{\rho}}} \gamma\left(n_{3}+k+i+1, \frac{3 u}{\sigma^{2} \bar{\rho}}\right),
\end{aligned}
$$

where $\gamma(.,$.$) is the lower incomplete gamma function.$ 


\section{APPENDIX C}

\section{SERIES REPRESENTATION OF (33)}

We define,

$$
\mathbf{M}\left(d_{1}, d_{2}, x\right) \triangleq \int_{0}^{\rho_{\text {th }}} u^{d_{1}} e^{-\frac{x}{3}} \gamma\left(d_{2}, x\right) d u .
$$

According to (50), the lower incomplete gamma function for integer-values first arguments, is:

$$
\gamma\left(d_{2}, x\right)=\Gamma\left(d_{2}\right)-\sum_{i=0}^{d_{2}-1} \frac{\Gamma\left(d_{2}\right)}{\Gamma(i+1)} x^{i} e^{-x} .
$$

Therefore,

$$
\begin{aligned}
& \mathbf{M}\left(d_{1}, d_{2}, x\right)=\int_{0}^{\rho_{\mathrm{th}}} u^{d_{1}} e^{-\frac{x}{3}}\left[\Gamma\left(d_{2}\right)-\sum_{i=0}^{d_{2}-1} \frac{\Gamma\left(d_{2}\right)}{\Gamma(i+1)} u^{i} e^{-u}\right] d u \\
& =\int_{0}^{\rho_{\mathrm{th}}} \Gamma\left(d_{2}\right) u^{d_{1}} e^{-\frac{x}{3}} d u-\sum_{i=0}^{d_{2}-1} \frac{\Gamma\left(d_{2}\right)}{\Gamma(i+1)} \int_{0}^{\rho_{\mathrm{th}}} u^{d_{1}+i} e^{-\frac{4 x}{3}} d u \\
& =3^{d_{1}+1} \Gamma\left(d_{2}\right) \gamma\left(d_{1}+1, \frac{\rho_{\mathrm{th}}}{3}\right) \\
& -\sum_{i=0}^{d_{2}-1} \frac{3^{d_{1}+i+1} \Gamma\left(d_{2}\right)}{4^{d_{1}+i+1} \Gamma(i+1)} \gamma\left(d_{1}+i+1, \frac{4 \rho_{\mathrm{th}}}{3}\right) .
\end{aligned}
$$

\section{REFERENCES}

[1] T. S. Rappaport, Y. Xing, G. R. MacCartney, A. F. Molisch, E. Mellios, and J. Zhang, "Overview of Millimeter Wave Communications for Fifth-Generation (5G) Wireless Networks With a Focus on Propagation Models," IEEE Transactions on Antennas and Propagation, vol. 65, no. 12, pp. 6213-6230, Dec 2017.

[2] S. Biswas, S. Vuppala, J. Xue, and T. Ratnarajah, "On the Performance of Relay Aided Millimeter Wave Networks," IEEE Journal of Selected Topics in Signal Processing, vol. 10, no. 3, pp. 576-588, 2016.

[3] S. Gong, C. Xing, Z. Fei, and S. Ma, "Millimeter-Wave Secrecy Beamforming Designs for Two-Way Amplify-and-Forward MIMO Relaying Networks," IEEE Transactions on Vehicular Technology, vol. 66, no. 3, pp. 2059-2071, 2017.

[4] Z. Wei, X. Zhu, S. Sun, Y. Huang, A. Al-Tahmeesschi, and Y. Jiang, "Energy-Efficiency of Millimeter-Wave Full-Duplex Relaying Systems: Challenges and Solutions," IEEE Access, vol. 4, pp. 4848-4860, 2016.

[5] N. Wei, X. Lin, and Z. Zhang, "Optimal Relay Probing in MillimeterWave Cellular Systems With Device-to-Device Relaying," IEEE Transactions on Vehicular Technology, vol. 65, no. 12, pp. 10218-10222, 2016.

[6] G. Yang, M. Xiao, H. Al-Zubaidy, Y. Huang, and J. Gross, "Analysis of millimeter-wave multi-hop networks with full-duplex buffered relays," IEEE/ACM Transactions on Networking (TON), vol. 26, no. 1, pp. 576590, 2018.

[7] S. Wu, R. Atat, N. Mastronarde, and L. Liu, "Improving the Coverage and Spectral Efficiency of Millimeter-Wave Cellular Networks Using Device-to-Device Relays," IEEE Transactions on Communications, vol. 66, no. 5, pp. 2251-2265, 2018.

[8] A. Chelli, K. Kansanen, M.-S. Alouini, and I. Balasingham, "On bit error probability and power optimization in multihop millimeter wave relay systems," IEEE Access, vol. 6, pp. 3794-3808, 2018.

[9] G. Yang and M. Xiao, "Performance Analysis of Millimeter-Wave Relaying: Impacts of Beamwidth and Self-Interference," IEEE Transactions on Communications, vol. 66, no. 2, pp. 589-600, 2018.

[10] G. Yang, M. Haenggi, and M. Xiao, "Traffic Allocation for LowLatency Multi-Hop Networks with Buffers," IEEE Transactions on Communications, vol. 66, no. 9, pp. 3999-4013, 2018.

[11] J. Zheng, J. Zhang, S. Chen, H. Zhao, and B. Ai, "Wireless powered UAV relay communications over fluctuating two-ray fading channels," Physical Communication, vol. 35, p. 100724, 2019.
[12] H. Hashemi, J. Haghighat, and M. Eslami, "Performance analysis of relay-aided millimeter-wave communications with optimal and suboptimal combining at destination," Physical Communication, vol. 39 p. 100991, 2020. [Online]. Available: http://www.sciencedirect.com/ science/article/pii/S1874490719304471

[13] G. D. Durgin, T. S. Rappaport, and D. A. de Wolf, "New analytical models and probability density functions for fading in wireless communications," IEEE Transactions on Communications, vol. 50, no. 6, pp. $1005-1015,2002$.

[14] M. Rao, F. J. Lopez-Martinez, M. Alouini, and A. Goldsmith, "MGF Approach to the Analysis of Generalized Two-Ray Fading Models," IEEE Transactions on Wireless Communications, vol. 14, no. 5, pp. 2548-2561, May 2015.

[15] J. M. Romero-Jerez, F. J. Lopez-Martinez, J. F. Paris, and A. J. Goldsmith, "The fluctuating two-ray fading model: Statistical characterization and performance analysis," IEEE Transactions on Wireless Communications, vol. 16, no. 7, pp. 4420-4432, 2017.

[16] A. A. Dos Anjos, T. R. R. Marins, C. R. Nogueira Da Silva, V. M. Rodrigo Penarrocha, L. Rubio, J. Reig, R. A. A. De Souza, and M. D. Yacoub, "Higher Order Statistics in a mmWave Propagation Environment," IEEE Access, vol. 7, pp. 103 876-103 892, 2019.

[17] T. S. Rappaport, G. R. MacCartney, M. K. Samimi, and S. Sun, "Wideband Millimeter-Wave Propagation Measurements and Channel Models for Future Wireless Communication System Design," IEEE Transactions on Communications, vol. 63, no. 9, pp. 3029-3056, Sept 2015.

[18] M. K. Samimi, G. R. MacCartney, S. Sun, and T. S. Rappaport, " $28 \mathrm{GHz}$ millimeter-wave ultrawideband small-scale fading models in wireless channels," in 2016 IEEE 83rd Vehicular Technology Conference (VTC Spring). IEEE, 2016, pp. 1-6.

[19] D. Wang, B. Bai, W. Chen, and Z. Han, "Achieving High Energy Efficiency and Physical-Layer Security in AF Relaying," IEEE Transactions on Wireless Communications, vol. 15, no. 1, pp. 740-752, 2016.

[20] J. Li, Z. Hu, and Y. Wang, "DF/AF Cooperative Relay in LTE-A," in 2010 IEEE 71st Vehicular Technology Conference, 2010, pp. 1-5.

[21] X. He and A. Yener, "Cooperation With an Untrusted Relay: A Secrecy Perspective," IEEE Transactions on Information Theory, vol. 56, no. 8, pp. 3807-3827, 2010

[22] Y. M. Khattabi and M. M. Matalgah, "Performance Analysis of MultipleRelay AF Cooperative Systems Over Rayleigh Time-Selective Fading Channels With Imperfect Channel Estimation," IEEE Transactions on Vehicular Technology, vol. 65, no. 1, pp. 427-434, 2016.

[23] H. A. Suraweera, H. K. Garg, and A. Nallanathan, "Performance Analysis of Two Hop Amplify-and-Forward Systems with Interference at the Relay," IEEE Communications Letters, vol. 14, no. 8, pp. 692-694, 2010.

[24] H. A. Suraweera, P. J. Smith, A. Nallanathan, and J. S. Thompson, "Amplify-and-Forward Relaying with Optimal and Suboptimal Transmit Antenna Selection," IEEE Transactions on Wireless Communications, vol. 10 , no. 6, pp. 1874-1885, 2011.

[25] H. A. Suraweera, T. A. Tsiftsis, G. K. Karagiannidis, and A. Nallanathan, "Effect of Feedback Delay on Amplify-and-Forward Relay Networks With Beamforming," IEEE Transactions on Vehicular Technology, vol. 60 , no. 3, pp. 1265-1271, 2011.

[26] G. Farhadi and N. C. Beaulieu, "On the performance of amplify-andforward cooperative systems with fixed gain relays," IEEE Transactions on Wireless Communications, vol. 7, no. 5, pp. 1851-1856, 2008.

[27] C. Wang, T. C. . Liu, and X. Dong, "Impact of Channel Estimation Error on the Performance of Amplify-and-Forward Two-Way Relaying," IEEE Transactions on Vehicular Technology, vol. 61, no. 3, pp. 1197-1207, 2012.

[28] H. Cui, M. Ma, L. Song, and B. Jiao, "Relay Selection for Two-Way Full Duplex Relay Networks With Amplify-and-Forward Protocol," IEEE Transactions on Wireless Communications, vol. 13, no. 7, pp. 37683777, 2014.

[29] L. Yang, K. Qaraqe, E. Serpedin, and M. Alouini, "Performance Analysis of Amplify-and-Forward Two-Way Relaying with Co-Channel Interference and Channel Estimation Error," IEEE Transactions on Communications, vol. 61, no. 6, pp. 2221-2231, 2013.

[30] V. A. Aalo, G. P. Efthymoglou, T. Soithong, M. Alwakeel, and S. Alwakeel, "Performance Analysis of Multi-Hop Amplify-and-Forward Relaying Systems in Rayleigh Fading Channels with a Poisson Interference Field," IEEE Transactions on Wireless Communications, vol. 13, no. 1, pp. 24-35, 2014.

[31] H. Hashemi, J. Haghighat, M. Eslami, and W. A. Hamouda, "Analysis of equal gain combining over fluctuating two-ray channels with 
applications to millimeter-wave communications," IEEE Transactions on Vehicular Technology, pp. 1-1, 2019.

[32] M. Olyaee, M. Eslami, and J. Haghighat, "Performance of maximum ratio combining of fluctuating two-ray (ftr) mmwave channels for $5 \mathrm{~g}$ and beyond communications," Transactions on Emerging Telecommunications Technologies, vol. 30, no. 10, p. e3601, 2019.

[33] H. Zhao, Z. Liu, and M. Alouini, "Different Power Adaption Methods on Fluctuating Two-Ray Fading Channels," IEEE Wireless Communications Letters, vol. 8, no. 2, pp. 592-595, April 2019.

[34] M. Olyaee, M. Eslami, H. Hashemi, and J. Haghighat, "Performance of Wireless Energy Transfer Efficiency in mm-Wave Communications," in 2019 27th Iranian Conference on Electrical Engineering (ICEE). IEEE, 2019, pp. 1611-1615.

[35] O. S. Badarneh and D. B. da Costa, "Cascaded fluctuating two-ray fading channels," IEEE Communications Letters, vol. 23, no. 9, pp. 1497-1500, 2019.

[36] H. Zhao, J. Zhang, L. Yang, G. Pan, and M.-S. Alouini, "Secure mmWave communications in cognitive radio networks," IEEE Wireless Communications Letters, vol. 8, no. 4, pp. 1171-1174, 2019.

[37] M. Bilim and N. Kapucu, "Average Symbol Error Rate Analysis of QAM Schemes Over Millimeter Wave Fluctuating Two-Ray Fading Channels," IEEE Access, vol. 7, pp. 105 746-105 754, 2019.

[38] D. Dixit and P. R. Sahu, "Performance of multihop detect-and-forward relaying system over fluctuating two-ray fading channels," Transactions on Emerging Telecommunications Technologies, vol. 29, no. 8, p. e3423, e3423 ett.3423. [Online]. Available: https://onlinelibrary.wiley.com/doi/ abs/10.1002/ett.3423

[39] J. Zhang, W. Zeng, X. Li, Q. Sun, and K. P. Peppas, "New results on the fluctuating two-ray model with arbitrary fading parameters and its applications," IEEE Transactions on Vehicular Technology, vol. 67, no. 3, pp. 2766-2770, 2017.

[40] M. O. Hasna and M.-S. Alouini, "Harmonic mean and end-to-end performance of transmission systems with relays," IEEE Transactions on communications, vol. 52, no. 1, pp. 130-135, 2004.

[41] O. Olabiyi and A. Annamalai, "Invertible exponential-type approximations for the Gaussian probability integral Q (x) with applications," IEEE Wireless Communications Letters, vol. 1, no. 5, pp. 544-547, 2012.

[42] I. Gradshteyn and I. Ryzhik, Table of Integrals, Series, and Products 2007 7th. USA Elsevier Edited by A. Jeffrey and D. Zwillinger, 2007.

[43] A. R. Miller and R. B. Paris, "Euler-type transformations for the generalized hypergeometric function $\mathrm{r}+2 \mathrm{~F} \mathrm{r}+1$ (x)," Zeitschrift für angewandte Mathematik und Physik, vol. 62, no. 1, pp. 31-45, 2011.

[44] A. Papoulis and S. U. Pillai, Probability, Random Variables, and Stochastic Processes, 4th ed. Boston: McGraw Hill, 2002. [Online]. Available: http://www.worldcat.org/search?qt=worldcat_org all\&q=0071226613 